\title{
Deregulation of the Genes that Are Involved in Drug Absorption, Distribution, Metabolism, and Excretion in Hepatocellular Carcinoma ${ }^{[\mathbf{S}}$
}

\author{
Dong Gui Hu, Shashikanth Marri, Ross A. McKinnon, Peter I. Mackenzie, and Robyn Meech \\ Department of Clinical Pharmacology and Flinders Centre for Innovation in Cancer (D.G.H., R.A.M., P.I.M., R.M.), and \\ Department of Molecular Medicine and Pathology (S.M.), Flinders University College of Medicine and Public Health, Flinders \\ Medical Centre, Bedford Park, South Australia, Australia
}

Received November 12, 2018; accepted December 19, 2018

\begin{abstract}
Genes involved in drug absorption, distribution, metabolism, and excretion (ADME) are called ADME genes. Currently, 298 genes that encode phase I and II drug metabolizing enzymes, transporters, and modifiers are designated as ADME genes by the PharmaADME Consortium. ADME genes are highly expressed in the liver and their levels can be influenced by liver diseases such as hepatocellular carcinoma (HCC). In this study, we obtained RNA-sequencing and microRNA (miRNA)-sequencing data from $371 \mathrm{HCC}$ patients via The Cancer Genome Atlas liver hepatocellular carcinoma project and performed ADME gene-targeted differential gene expression analysis and expression correlation analysis. Two hundred thirty-three of the 298 ADME genes $(78 \%)$ were expressed in HCC. Of these genes, almost one-quarter (58 genes) were significantly downregulated, while only $6 \%$ (15) were upregulated in HCC relative to healthy liver. Moreover, one-half (14/28) of the core
\end{abstract}

ADME genes (CYP1A2, CYP2A6, CYP2B6, CYP2C8, CYP2C9, CYP2C19, CYP2E1, CYP3A4, NAT1, NAT2, UGT2B7, SLC22A1, $S L C O 1 B 1$, and SLCO1B3) were downregulated. In addition, about one-half of the core ADME genes were positively correlated with each other and were also positively ( $A H R$, ARNT, HNF4A, PXR, CAR, PPARA, and RXRA) or negatively (PPARD and PPARG) correlated with transcription factors known as ADME modifiers. Finally, we show that most miRNAs known to regulate core ADME genes are upregulated in HCC. Collectively, these data reveal 1) an extensive transcription factor-mediated ADME coexpression network in the liver that efficiently coordinates the metabolism and elimination of endogenous and exogenous compounds; and 2) a widespread deregulation of this network in HCC, most likely due to deregulation of both transcriptional and post-transcriptional (miRNA) pathways.

\section{Introduction}

The liver expresses a variety of phase I and II enzymes and transporters and thus it is the primary organ of drug and xenobiotic metabolism and clearance. Phase I enzymes mainly perform oxidative, reductive, or hydrolytic reactions that usually create or reveal a functional group (e.g., hydroxyl, carboxyl, and amino) on a xenobiotic, whereas phase II enzymes generally add a water-soluble group (e.g., glutathione, sulfate, and glucuronic acid) directly to a xenobiotic or to a

R.A.M. is a Cancer Council/SA Health Beat Cancer Professorial Chair During the preparation period, P.I.M. was a National Health and Medical Research Council Senior Principal Research Fellow and R.M. was an Australian Research Council Future Fellow.

This study was supported by the National Health and Medical Research Council of Australia [Grant ID 1143175 (to R.M., R.A.M., P.I.M., and D.G.H.), Grants ID1020931 (to P.I.M.) and ID1085410 (to P.I.M., R.A.M., and R.M.)]. The project was also supported by funding from the Flinders Medical Centre Foundation.

https://doi.org/10.1124/jpet.118.255018.

S This article has supplemental material available at jpet.aspetjournals.org. metabolite of phase I metabolism. The influx and efflux transporters are responsible for the uptake and excretion of various compounds (e.g., drugs) in and out of cells. The group of genes involved in drug absorption, distribution, metabolism, and excretion (ADME) consist mainly of genes encoding phase I and II drug metabolizing enzymes, drug transporters, and modifiers; the latter are a group of factors that either modulate the expression of other ADME genes or influence the biochemistry of ADME enzymes (www.pharmaadme.org). Currently, 298 genes are classified as ADME genes by the pharmaADME Consortium, including 32 core ADME genes and 266 extended ADME genes (www.pharmaadme.org). The combined activities of the ADME genes determine the capacity of the liver to metabolize and clear drugs and hence influence the efficacy of drug treatment.

The expression and activities of ADME genes are influenced by liver diseases, such as viral infection, alcohol liver disease, primary sclerosis cholangitis, nonalcoholic fatty liver disease, and hepatocellular carcinoma (HCC) (Kurzawski et al., 2012;

ABBREVIATIONS: ABC, ATP-binding cassette; ADH, alcohol dehydrogenase; ADME, absorption, distribution, metabolism, and excretion; AKR, aldo-keto reductases; ALDH, aldehyde dehydrogenase; CAR, constitutive androstane receptor; GST, glutathione S-transferase; HCC, hepatocellular carcinoma; HT-seq, high-throughput sequencing; LIHC, liver hepatocellular carcinoma; miRNA, microRNA; miRNA-seq, microRNA sequencing; P450, cytochrome P450; PXR, pregnane X receptor; RNA-seq, RNA sequencing; SLC, solute carrier; SULT, sulfotransferase; TCGA, The Cancer Genome Atlas; TF, transcription factor; UGT, UDP-glucuronosyltransferase. 
Hardwick et al., 2013). For example, nonalcoholic fatty liver disease significantly alters the expression of ADME genes that encode a variety of phase I and II drug metabolizing enzymes and drug transporters, including cytochrome P450 (P450) enzymes (Fisher et al., 2009), glutathione $S$-transferases (GSTs) (Hardwick et al., 2010), UDP-glucuronosyltransferases (UGTs) and sulfotransferases (SULTs) (Congiu et al., 2002; Hardwick et al., 2013), and ATP-binding cassette (ABC) transporters (Hardwick et al., 2011).

HCC is the fifth most common cancer and the third highest cause of cancer-related death globally (Lin et al., 2012). Common risk factors for HCC are viral infection, chronic alcohol consumption, aflatoxin exposure, and cirrhosis (Farazi and DePinho, 2006). Surgical resection, liver transplantation, and ablation remain the only potentially curative treatments for early stage HCC, whereas two multikinase inhibitors, sorafenib and regorafenib, are the only approved systemic drugs for treating advanced HCC (Llovet et al., 2008; Bruix et al., 2017). The sequential use of sorafenib (front line) and regorafenib (second line) provides a survival benefit in advanced HCC (Bruix et al., 2017).

Several studies have investigated the differential expression of ADME genes in HCC relative to adjacent noncancerous liver tissue. Many of these studies reported altered expression of a single ADME gene or gene superfamily, such as P450s (Chen et al., 2014; Yan et al., 2015a,b), ABC transporters (Borel et al., 2012), or UGTs (Yan et al., 2015a). Some highthroughput studies using microarray-based gene expression profiling (Okabe et al., 2001; Lee et al., 2004; Park et al., 2006; Jin et al., 2015a), or more recently deep RNA sequencing (RNA-seq) (Ho et al., 2015; Woo et al., 2017), have revealed altered expression of ADME genes from a number of superfamilies (e.g., P450s, UGTs, and GSTs); however, these untargeted studies did not draw specific conclusions about dysregulation of ADME pathways. Based on the limitations of these previous studies, we sought to provide the first targeted, yet comprehensive, dysregulation analysis of all known ADME genes in HCC.

The Cancer Genome Atlas (TCGA) liver hepatocellular carcinoma (LIHC) project contains RNA-seq data from $371 \mathrm{HCC}$ patients including 50 paired HCC and corresponding adjacent noncancerous liver tissues (https://portal.gdc.cancer. gov/). In the present study, we performed differential gene expression analysis of the 50 paired HCC and noncancerous tissues focusing on the defined ADME gene set. Key findings were that one-half of the core ADME gene set and about one-quarter of the extended ADME set were significantly downregulated in HCC, while few genes were upregulated. Furthermore, about one-half of the core ADME genes showed significant correlation with each other and were also significantly correlated with nine transcription factors (TFs) [AHR, ARNT, HNF4A, pregnane $\mathrm{X}$ receptor (PXR), constitutive androstane receptor (CAR), PPARA, PPARD, PPARG, and RXRA], indicating coordinated regulation at the transcriptional level. The study also identified specific microRNAs (miRNAs) likely to be involved in ADME gene dysregulation in HCC.

\section{Materials and Methods}

Liver Hepatocellular Carcinoma Data Set of TCGA. The Cancer Genome Atlas is a community resource project that collects and analyzes cancer specimens from a variety of human cancers (https://cancergenome.nih.gov). Data generated from TCGA projects such as RNA-seq and miRNA sequencing (miRNA-seq) for various human cancers are made freely available for browser, download, and use for commercial, scientific, and educational purposes. The results shown in the present study were mainly based on RNA-seq and miRNA-seq data generated by the TCGA Research Network of the LIHC project (https://portal.gdc.cancer.gov/projects/TCGA-LIHC). The TCGA LIHC project contains RNA-seq data of HCC tumors from 371 patients. RNA-seq data from normal liver tissues adjacent to the corresponding tumors are also available for 50 of these patients. The TCGA LIHC project also contains miRNA-seq data for these patients, including paired normal and cancerous specimens from 49 patients. As described in detail subsequently, the RNA-seq and miRNA-seq data from the paired normal and cancerous specimens were used for differential gene and miRNA expression analysis, respectively, and the RNA-seq data from the 371 tumors were used for gene expression correlation analysis.

The detailed clinical data for each of the 371 HCC patients are available from the cBioportal (http://www.cbioportal.org/study? id=lihc_tcga\#clinical). This cohort includes 250 male and 121 female patients with the age at diagnosis ranging from 16 to 90 years. The ethnicities of the subjects are available for 361 patients, including Asians (158), Caucasians (184), Black or African Americans (17), and American Indian or Alaska Native (2). The American Joint Committee on Cancer proposes a staging grouping based on $\mathrm{T}, \mathrm{N}$, and $\mathrm{M}$ to define the three stages of tumors: the primary tumor $(\mathrm{T})$, regional lymph nodes $(\mathrm{N})$, and distant metastases (M) (Kamarajah et al., 2018). The progression stages of the tumors are available for 368 patients. According to this TNM staging system, all tumors were primary hepatocellular carcinomas at stage $\mathrm{T}$, including $181,94,80$, and 13 tumors at stages $\mathrm{T} 1, \mathrm{~T} 2, \mathrm{~T} 3$, and T4, respectively. Tumors at stages $\mathrm{N}$ and $\mathrm{M}$ were not included in the TCGA LIHC project. The TCGA LIHC clinical data do not provide the details of the pre/postoperative chemotherapeutic regimens of the patients.

ADME Genes Defined by the PharmaADME Consortium. Thirty-two core and 266 extended ADME genes (a total of 298 genes) are currently defined by the PharmaADME Consortium (http://www. pharmaadme.org). The core ADME gene set represents the most important genes directly involved in drug metabolism/clearance; the extended $\mathrm{ADME}$ gene set represents other genes related to drug metabolism/clearance. Four extended ADME genes, either pseudogene (CYP2D7P1), antisense gene (SLC22A18AS), or functionally unknown genes (LOC728667 and LOC731931) were excluded from analysis in this study. The remaining 294 ADME genes (32 core; 262 extended) are listed in Supplemental Table 1.

Download of the TCGA LIHC RNA-Seq and miRNA-Seq Data. For differential gene expression analysis, TCGA LIHC RNAseq data from the 50 paired HCC and corresponding adjacent noncancerous liver tissues that have been mapped to the human genome GRCh38 reference assembly were downloaded as high-throughput sequencing (HT-seq) counts from the Genomic Data Commons Data Portal (https://portal.gdc.cancer.gov/). The HT-seq counts contained the raw read counts of each gene generated using a HT-seq tool (http:// htseq.readthedocs.io/en/release_0.9.1/) to measure gene expression level from the Genomic Data Commons mRNA quantification analysis pipeline (https://docs.gdc.cancer.gov/Data/Bioinformatics_Pipelines/ Expression_mRNA_Pipeline/). The HT-seq counts of 609 genes that were included in the analysis in this study are given in Supplemental Table 2 .

For gene expression correlation analysis, the TCGA LIHC RNA-seq data from 371 HCC tumors were downloaded in the form of HT-seq counts from the Genomic Data Commons Data Portal (https://portal. gdc.cancer.gov/). Genes (protein coding and noncoding) with a mean of less than 10 counts were discarded; the counts of the remaining genes were normalized using the upper quantile normalization method. The normalized read counts of the 609 genes that were included in the analysis of this study are given in Supplemental Table 3. 
For differential miRNA expression analysis, TCGA LIHC miRNAseq data of the 49 paired HCC and corresponding adjacent noncancerous liver tissues were downloaded from The Cancer Genome Atlas data portal (https://portal.gdc.cancer.gov/) and normalized using the upper quantile normalization method. The normalized read counts of miRNAs that were included in the analysis of this study are given in Supplemental Table 4.

ADME Genes and Non-ADME Genes Analyzed in this Study. Most of the 298 ADME genes belong to gene superfamilies such as ABC transporters, P450 enzymes, solute carrier (SLC) transporters, and UGT enzymes. However, many of these gene superfamilies also contain members that are not involved in drug absorption, distribution, metabolism, and excretion. These genes are termed non-ADME genes in this study. The TCGA LIHC RNA-seq data showed HT-seq counts for 743 genes, including 290 ADME genes and 453 non-ADME genes. For quality control, genes with HT-seq counts of $<32$ (equivalent to $<5$ of $\log 2$-transformed counts) were excluded from the analysis. Based on this cutoff criterion, a total of 609 genes (Supplemental Table 2), including 233 ADME genes and 376 non-ADME genes, were included in the final analysis. Inclusion of the non-ADME genes in our analysis allowed us to determine whether ADME genes were specifically dysregulated, rather than whole gene superfamilies.

Statistical Analysis of the TCGA LIHC RNA-Seq Data and miRNA-Seq Data. The downloaded RNA-seq (HT-seq counts) of the 50 paired HCC and corresponding adjacent normal liver tissues as described previously were subjected to differential gene expression analysis using the DESeq2 program (Love et al., 2014). Briefly, DESeq2 takes the HT-seq counts per gene and normalizes gene counts using a generalized linear model. After normalization, it estimates negative binomial distribution and performs variance shrinkage to determine the fold changes of genes between normal and cancerous tissues. DESeq2 uses a Wald test for statistically significant testing. Briefly, the shrunken estimate of log fold change is divided by its S.E., resulting in a $z$-statistic, which is compared with a standard normal distribution. The Wald test allows testing of individual coefficients, or contrasts of coefficients, without the need to fit a reduced model as with the likelihood ratio test. The Wald test $P$ values from the subset of genes that pass the independent filtering step are adjusted for multiple testings using the BenjaminiHochberg test. As detailed in Supplemental Table 5, the final report of the DESeq 2 analysis included a $\log 2$ fold change in the expression level of each gene between the tumor and adjacent nontumor liver tissues and the associated S.E. estimate for the log 2 fold change estimate and the Wald test result ( $P$ value) and the BenjaminiHochberg test results (adjusted $P$ values). An adjusted $P$ value of $<0.05$ was considered statistically significant. Differentially expressed genes were defined by a $\log 2$ fold change of $>1$ (equivalent to an upregulation of $>2$-fold) or $<-1$ (equivalent to a downregulation of $<2$-fold). Supplemental Table 5 gives the final results pertaining to the 609 genes that are included in analysis in this study.

The normalized gene expression levels (read counts) of the 371 HCC tumors as described previously were subjected to Spearman ranking correlation analysis 1) between core $\mathrm{ADME}$ genes and 2) between core ADME genes and nine ADME modifier transcription factors and NRF2 and its coregulatory KEAP1 using GraphPad Prism (version 7.03). A $P$ value of $<0.05$ was considered statistically significant.

The normalized expression levels of miRNAs of the 49 paired HCC and corresponding adjacent normal noncancerous liver tissues as described previously were analyzed using a paired $t$ test via GraphPad Prism (version 7.03 GraphPad, LA Jolla, CA). A $P$ value of $<0.05$ was considered statistically significant.

Analysis of Microarray-Based Gene Expression Profiles of Hepatocellular Carcinoma. Most of the results reported in this study were obtained from the analysis of the TCGA LIHC RNA-seq data and miRNA-seq data as described previously. To support the findings from this data set, we further analyzed microarray-based whole genome gene expression profiles from four other studies (Chen et al., 2002; Wurmbach et al., 2007; Roessler et al., 2010) via Oncomine, a publicly accessible database (Rhodes et al., 2007). As stated by Oncomine (www.oncomine.org), Roessier et al. (2010) analyzed $225 \mathrm{HCC}$ and 220 adjacent nontumor liver tissues using the Affymetrix human genome 96 HT HG-U133A 2.0 microarray platform (GSE14520). Using the same U133A 2.0 microarray, Wurbach et al. (2007) studied 35 HCC tumors and 10 heathy liver tissues (GSE6764). Chen et al. (2002) analyzed HCC tumor liver tissues (71-76 specimens) and adjacent nontumor liver tissues (98-104 specimens) using custom-made cDNA microarrays containing 23,075 cDNA probes, representing 17,400 genes. Thurnherr et al. (2016) studied 100 paired HCC tumor and adjacent nontumor liver tissues using Agilent's 60-mer oligo microarray (Agilent Technologies, Santa Clara, CA) (GSE62043).

\section{Results}

Deregulation of ADME and Non-ADME Genes in HCC. This study examined the expression of 233 ADME genes (Supplemental Table 6) in 50 paired HCC and corresponding noncancerous liver tissues using the TCGA LIHC RNA-seq data set. Three hundred seventy-six non-ADME genes from ADME gene superfamilies that are not involved in drug metabolism and clearance were also analyzed for comparison. Overall, of the 609 genes analyzed, 109 genes (18\%) were downregulated (Supplemental Table 7) and 55 genes (9\%) were upregulated (Supplemental Table 8) in HCC. Among the 233 ADME genes, 58 genes (24\%) were downregulated (Supplemental Table 9) and 15 genes (6\%) were upregulated (Supplemental Table 10) in HCC, whereas out of the 376 non-ADME genes, 51 genes (14\%) were downregulated (Supplemental Table 11) and 40 genes (11\%) were upregulated (Supplemental Table 12) in HCC. Chi-square tests showed that ADME genes were significantly more likely to be downregulated than upregulated $(P<0.0001)$, and that ADME genes were significantly more likely to be downregulated than non-ADME genes $(P<0.001)$.

Deregulation of Core ADME Genes in HCC. The PharmaADME Consortium (www.pharmaadme.org) currently defines 32 core ADME genes (Supplemental Table 13), four (GSTT1, SLC15A2, SLC22A2, and SLC22A6) of which were not expressed in the TCGA HCC specimens. Of the remaining 28 core ADME genes, 14 (50\%) were significantly downregulated in HCC tissues compared with paired adjacent noncancerous liver tissues, including eight phase I enzymes (CYP1A2, CYP2A6, CYP2B6, CYP2C8, CYP2C9, CYP2C19, CYP2E1, and CYP3A4), three phase II enzymes (NAT1, NAT2, and UGT2B7), and three transporters (SLC22A1, SLCO1B1, and SLCO1B3) (Fig. 1; Table 1). None of the core ADME genes were upregulated. Chi-square tests showed that core ADME genes were significantly more likely to be downregulated relative to extended ADME genes $(P<0.01)$ or non-ADME genes $(P<0.0001)$. Our analyses of four other microarray gene expression profiling studies of HCC (Chen et al., 2002; Wurmbach et al., 2007; Roessler et al., 2010; Thurnherr et al., 2016) showed that each of the 14 downregulated core ADME genes was reported to be downregulated in HCC in at least one of these other studies (Table 2).

Deregulation of ADME Genes Coding for Phase I Drug Metabolism Enzymes in HCC. One hundred and thirty of the 298 ADME genes code for phase I drug 


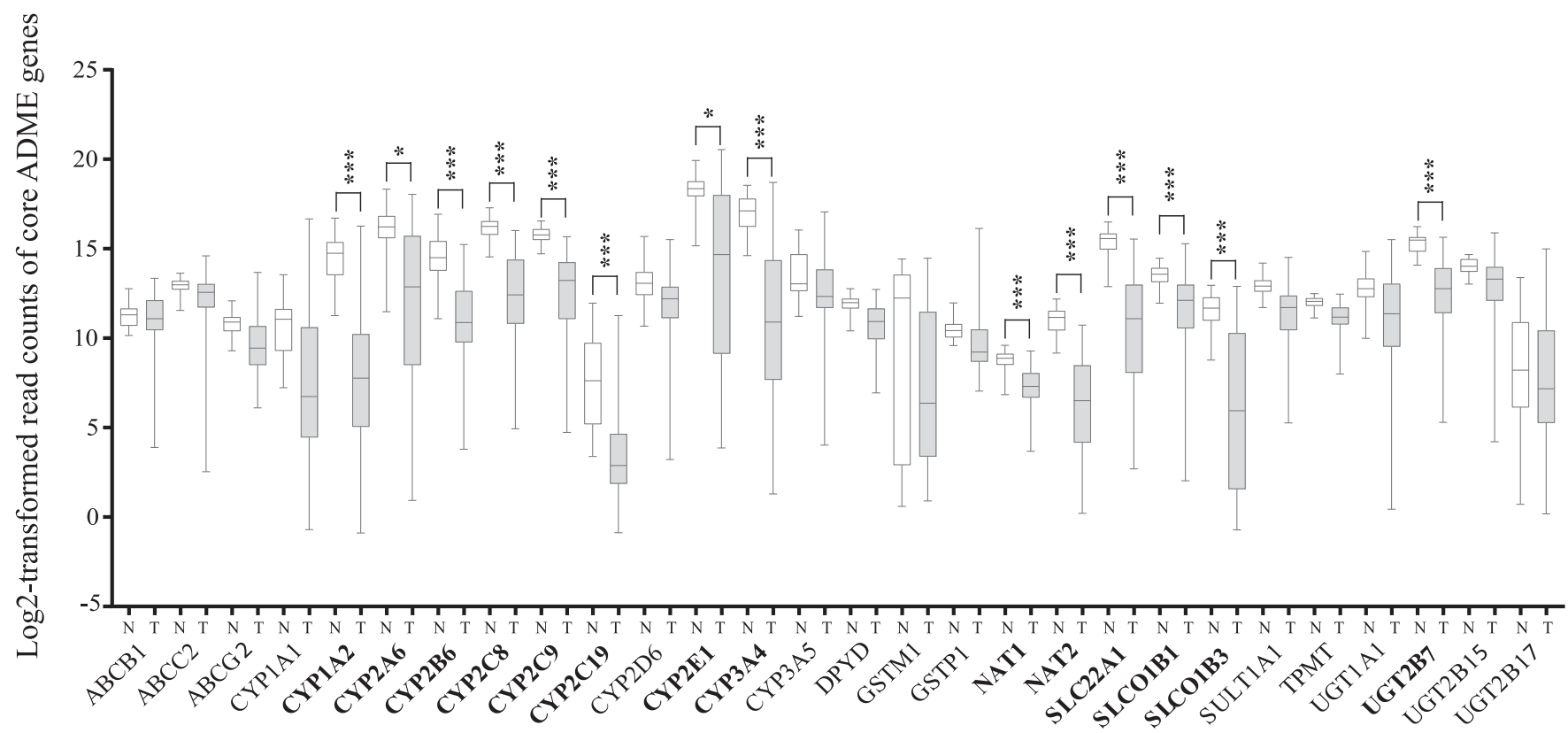

Fig. 1. Deregulation of core ADME genes in HCC. The TCGA LIHC RNA-seq data set representing 50 paired HCC cancerous and corresponding adjacent noncancerous liver tissues was downloaded from the Genomic Data Commons Data Portal (https://portal.gdc.cancer.gov/) and subjected to differential gene expression analysis using the DESeq2 program as described in Materials and Methods. The box-and-whisker plot shows the log 2 -transformed expression levels (minimum, first quartile, median, third quartile, and maximum) of 28 core ADME genes in $50 \mathrm{HCC}$ cancer tissues and their corresponding adjacent noncancerous liver tissues. ${ }^{*} P<0.05 ; * * * P<0.001 . \mathrm{N}$, noncancerous liver tissues; T, HCC cancerous liver tissues.

metabolizing enzymes (PharmaADME). Of these genes, $106(82 \%)$ were expressed in the TCGA HCC specimens, including 34 downregulated genes (32\%) (Table 1) and five upregulated genes (5\%): ALDH3A1, CBR3, CYP17A1, NOS2A, and $C Y P 7 A 1$. Among the downregulated genes, 27 encode members of three enzyme superfamilies, cytochromes P450 enzymes, alcohol dehydrogenases (ADHs), and aldehyde dehydrogenases (ALDHs). The remaining seven genes were $A O X 1$, DHRS1, EPHX2, PLGLB1, PON1, PON3, and XDH.

There are 57 putatively functional CYP genes in the human genome that are grouped into 18 families and 44 subfamilies based on sequence similarity (Nelson et al., 2004). Forty-seven CYP genes are classified as ADME genes (PhamaADME). Of these ADME CYP genes, 37 were expressed in the TCGA HCC specimens: two of these were upregulated (CYP7A1 and CYP17A1) and 17 were downregulated (Fig. 2A; Table 1). The downregulated CYP genes included the eight aforementioned downregulated core ADME CYP genes (Fig. 1) and nine other CYP genes (CYP2C18, CYP3A7, CYP3A43, CYP4A11, CYP4F2, CYP4F12, CYP8B1, CYP26A1, and CYP39A1).

The human genome has seven functional $A D H$ genes ( $1 \mathrm{~A}$, $1 \mathrm{~B}, 1 \mathrm{C}, 4,5,6$, and 7) that are all classified as ADME genes based on their roles in drug clearance (Edenberg, 2007). Of these $A D H$ genes, six $(1 \mathrm{~A}, 1 \mathrm{~B}, 1 \mathrm{C}, 4,5$, and 6$)$ were expressed in the TCGA HCC specimens, five of which (1A, $1 \mathrm{~B}, 1 \mathrm{C}, 4$, and 6 ) were significantly downregulated in HCC, consistent with the findings of recent reports (Wei et al., 2012; Shang et al., 2017).

There are $19 A L D H$ genes in humans (Marchitti et al., 2008), 15 of which are classified as ADME genes (www. pharmaadme.org). Of the ADME $A L D H$ genes, 14 were expressed in the TCGA HCC specimens, including five genes that were downregulated ( $A L D H 1 A 3,1 B 1,2,6 A 1$, and $8 A 1)$ and one that was upregulated $(A L D H 3 A 1)$ in HCC (Fig. 2B;
Table 1). The downregulation of $A L D H 1 B 1$ (Yang et al., 2017) and $A L D H 2$ (Jin et al., 2015b) in HCC was consistent with recent reports, whereas the downregulation of $A L D H 1 A 3$, $A L D H 6 A 1$, and $A L D H 8 A 1$ and the upregulation of $A L D H 3 A 1$ in $\mathrm{HCC}$ were novel findings.

Deregulation of ADME Genes Coding for Phase II Drug Metabolism Enzymes in HCC. Sixty-eight of the 298 ADME genes encode for phase II drug metabolizing enzymes (PharmaADME). Of these ADME genes, $52(76 \%)$ were expressed in the TCGA HCC specimens, including 11 genes $(23 \%)$ that were downregulated and three genes $(6 \%)$ that were upregulated in HCC. Seven downregulated genes encode for members of three enzyme superfamilies (GSTs, SULTs, and UGTs), as described in detail subsequently, and the other four genes were carbohydrate (N-acetylglucosamine 6-O) sulfotransferase 4 (CHST4), nicotinamide $\mathrm{N}$-methyltransferase (NNMT), and two arylamine $\mathrm{N}$-acetyltransferases (NAT1 and NAT2). The upregulated genes were CHST10, SULT1C2, and UGT2B11.

The human genome contains 25 GST genes (www.genenames.org), 21 of which are classified as ADME genes (www. pharmaadme.org). Of these ADME GST genes, 17 genes from three GST subfamilies (cytosolic, mitochondrial, and microsomal) were expressed in the TCGA HCC specimens, including two GST genes (GSTM5 and GSTZ1) that were significantly downregulated in HCC (Fig. 3A). GSTZ1 downregulation in HCC was recently reported (Jahn et al., 2016). The downregulation of GSTA1 (Li et al., 2008) and GSTP1 (Zhong et al., 2002; Zhang et al., 2005; Shen et al., 2012) and the upregulation of GST1A4 (Liu et al., 2017) in HCC were also previously reported. There was no overall significant deregulation of these three GST genes in HCC, but we observed reduced GSTA1 and GSTP1 mRNA levels and increased GST1A4 mRNA levels in HCC tumor tissues 
TABLE 1

Twenty-eight core ADME genes and 205 extended ADME genes that are expressed in TCGA HCC cohort and their deregulation in HCC tumor tissues compared with corresponding adjacent noncancerous liver tissues

Downregulated genes are bold and upregulated genes are bold with an asterisk.

\begin{tabular}{|c|c|c|c|}
\hline Phase I Enzyme & Phase II Enzyme & Transporter & Modifier \\
\hline \multicolumn{4}{|l|}{ Core ADME Genes } \\
\hline CYP1A1 & GSTM1 & ABCB1 & \\
\hline CYP1A2 & GSTP1 & $\mathrm{ABCC} 2$ & \\
\hline CYP2A6 & NAT1 & ABCG2 & \\
\hline CYP2B6 & NAT2 & SLC22A1 & \\
\hline CYP2C19 & SULT1A1 & SLCO1B1 & \\
\hline CYP2C8 & TPMT & SLCO1B3 & \\
\hline CYP2C9 & UGT1A1 & & \\
\hline CYP2D6 & UGT2B15 & & \\
\hline CYP2E1 & UGT2B17 & & \\
\hline CYP3A4 & UGT2B7 & & \\
\hline \multicolumn{4}{|l|}{ CYP3A5 } \\
\hline \multicolumn{4}{|l|}{ DPYD } \\
\hline \multirow{2}{*}{\multicolumn{4}{|c|}{ Extended ADME Genes }} \\
\hline ADH1A, CYP51A1 & CHST1 & & AHR \\
\hline ADH1B, CYP7A1* & CHST10* & ABCA4* & ARNT \\
\hline ADH1C, CYP7B1 & CHST11 & ABCB11 & ARSA \\
\hline ADH4, CYP8B1 & CHST12 & $\mathrm{ABCB} 4$ & ATP7A \\
\hline $\mathrm{ADH} 5, \mathrm{DDO}$ & CHST13 & ABCB6 & ATP7B \\
\hline ADH6, DHRS1 & CHST2 & ABCB7 & CAT \\
\hline ADHFE1, DHRS12 & CHST3 & ABCB8 & CDA \\
\hline ALDH1A1, DHRS13 & CHST4 & ABCC1 & HNF4A \\
\hline ALDH1A2, DHRS2 & CHST7 & ABCC10* & KCNJ11* \\
\hline ALDH1A3, DHRS3 & CHST9 & ABCC11 & MAT1A \\
\hline ALDH1B1, DHRS4 & GSTA1 & ABCC3 & NR1I2 \\
\hline ALDH2, DHRS4L1 & GSTA2 & ABCC4* & NR1I3 \\
\hline ALDH3A1*, DHRS4L2 & GSTA4 & $\mathrm{ABCC} 6$ & POR \\
\hline ALDH3A2, DHRS7 & GSTCD & ABCC9 & PPARA \\
\hline ALDH3B1, DHRS7C & GSTK1 & ABCG1 & PPARD \\
\hline ALDH4A1, DHRS9 & GSTM2 & SLC10A1 & PPARG \\
\hline ALDH5A1, DHRSX & GSTM3 & SLC13A2 & RXRA \\
\hline ALDH6A1, EPHX1 & GSTM4 & SLC13A3 & SERPINA7 \\
\hline ALDH7A1, EPHX2 & GSTM5 & SLC15A1 & SOD1 \\
\hline ALDH8A1, FMO1 & GSTO1 & SLC16A1 & SOD2 \\
\hline ALDH9A1, FMO3 & GSTO2 & SLC19A1 & SOD3 \\
\hline AOX1, FMO4 & GSTZ1 & SLC22A10 & \\
\hline CBR1, FMO5 & HNMT & SLC22A11* & \\
\hline CBR3*, GPX1 & MGST1 & SLC22A15* & \\
\hline CES1, GPX2 & MGST2 & SLC22A17 & \\
\hline CES2, GPX3 & MGST3 & SLC22A18 & \\
\hline CYB5R3, GPX4 & NNMT & SLC22A3 & \\
\hline CYP11A1, GPX7 & SULF1 & SLC22A4* & \\
\hline CYP17A1*, GSR & SULT1A2 & SLC22A5 & \\
\hline CYP1B1, GSS & SULT1B1 & SLC22A7 & \\
\hline CYP20A1, HAGH & SULT1C1 & SLC22A9 & \\
\hline CYP21A2, HSD11B1 & SULT1C2* & SLC27A1 & \\
\hline CYP26A1, HSD17B11 & SULT1E1 & SLC28A1 & \\
\hline CYP27A1, HSD17B14 & SULT2A1 & SLC29A1 & \\
\hline CYP2A13, METAP1 & UGT1A3 & SLC29A2 & \\
\hline CYP2A7, NOS1 & UGT1A4 & SLC2A4 & \\
\hline CYP2C18, NOS2A* & UGT1A6 & SLC5A6 & \\
\hline CYP2J2, NOS3 & UGT1A9 & SLC7A5 & \\
\hline CYP2R1, PDE3A & UGT2A1 & SLC7A7 & \\
\hline CYP2S1, PDE3B & UGT2B10 & SLC7A8 & \\
\hline CYP39A1, PLGLB1 & UGT2B11* & SLCO1A2 & \\
\hline CYP3A43, PON1 & UGT2B4 & SLCO2A1* & \\
\hline CYP3A7, PON2 & & SLCO2B1 & \\
\hline CYP46A1, PON3 & & SLCO3A1 & \\
\hline CYP4A11, XDH & & SLCO4A1 & \\
\hline CYP4F11 & & SLCO4C1 & \\
\hline CYP4F12 & & TAP1 (ABCB2) & \\
\hline CYP4F2 & & TAP2 (ABCB3) & \\
\hline CYP4F3 & & & \\
\hline
\end{tabular}

compared with corresponding adjacent normal liver tissues in nearly $50 \%$ of the patients (data not shown).

There are 13 SULT genes in the human genome, 10 of which are classified as ADME genes (Blanchard et al., 2004). Of these $S U L T$ genes, six (1A1,1A2,1B1, 1C2, 1E1, and 2A1) were expressed in the TCGA HCC specimens, including three genes that were downregulated (1A2, 1E1, and 2A1) and one gene that was upregulated (1C2) in HCC (Fig. 3B). In 
TABLE 2

Downregulation of 14 core ADME genes in hepatocellular carcinoma

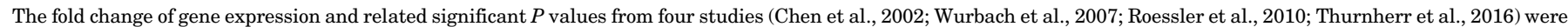

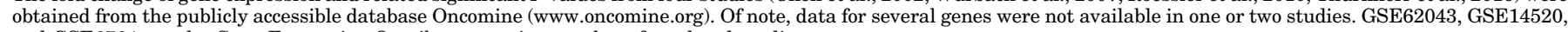
and GSE6764 are the Gene Expression Omnibus accession numbers for related studies.

\begin{tabular}{|c|c|c|c|c|c|c|c|c|c|c|}
\hline \multirow{2}{*}{ Gene } & \multicolumn{2}{|c|}{$\begin{array}{l}\text { TCGA HCC [This Study; } \\
\text { T }(50) / \mathrm{N}(50)]\end{array}$} & \multicolumn{2}{|c|}{$\begin{array}{c}\text { Thurnherr et al. (2016) } \\
\text { [T (100)/N (100); GSE62043] }\end{array}$} & \multicolumn{2}{|c|}{$\begin{array}{c}\text { Roessler et al. (2010) [T (225)/ } \\
\text { N (220); GSE14520] }\end{array}$} & \multicolumn{2}{|c|}{$\begin{array}{l}\text { Wurmbach et al. (2007) } \\
\text { [T (35)/N (10); GSE6764] }\end{array}$} & \multicolumn{2}{|c|}{$\begin{array}{l}\text { Chen et al. }(2002) \\
{[\mathrm{T}(98-104) / \mathrm{N}(71-76)]}\end{array}$} \\
\hline & $\begin{array}{l}\text { Fold } \\
\text { Change }\end{array}$ & $P$ Value & $\begin{array}{l}\text { Fold } \\
\text { Change }\end{array}$ & $P$ Value & $\begin{array}{l}\text { Fold } \\
\text { Change }\end{array}$ & $P$ Value & $\begin{array}{l}\text { Fold } \\
\text { Change }\end{array}$ & $P$ Value & $\begin{array}{l}\text { Fold } \\
\text { Change }\end{array}$ & $P$ Value \\
\hline CYP1A2 & -5.79 & $8.05 \times 10^{-7}$ & -7.25 & $1.06 \times 10^{-33}$ & -18.87 & $1.50 \times 10^{-131}$ & -23.814 & $1.69 \times 10^{-9}$ & & \\
\hline CYP2A6 & -2.08 & 0.021 & -6.07 & $3.35 \times 10^{-19}$ & -11.33 & $7.21 \times 10^{-72}$ & & & -6.53 & $6.56 \times 10^{-15}$ \\
\hline CYP2B6 & -5.68 & $1.26 \times 10^{-15}$ & -1.52 & $4.85 \times 10^{-21}$ & -10.80 & $3.59 \times 10^{-100}$ & & & -2.95 & $9.51 \times 10^{-27}$ \\
\hline CYP2C8 & -5.40 & $6.67 \times 10^{-17}$ & -7.88 & $5.10 \times 10^{-34}$ & & & -3.561 & $2.69 \times 10^{-5}$ & -5.76 & $2.32 \times 10^{-20}$ \\
\hline CYP2C9 & -4.09 & $1.75 \times 10^{-13}$ & -5.11 & $1.48 \times 10^{-14}$ & -4.87 & $2.89 \times 10^{-95}$ & & & -5.60 & $1.33 \times 10^{-19}$ \\
\hline CYP2C19 & -6.24 & $6.06 \times 10^{-8}$ & & & -4.87 & $1.15 \times 10^{-67}$ & & & & \\
\hline CYP2E1 & -2.03 & 0.028 & -4.34 & $4.43 \times 10^{-17}$ & -8.69 & $3.46 \times 10^{-38}$ & -3.825 & $2.48 \times 10^{-5}$ & & \\
\hline CYP3A4 & -3.32 & 0.00027 & -4.00 & $7.87 \times 10^{-14}$ & -7.09 & $3.85 \times 10^{-71}$ & & & -2.53 & $3.17 \times 10^{-10}$ \\
\hline NAT1 & -2.37 & $2.10 \times 10^{-15}$ & -1.59 & $1.11 \times 10^{-13}$ & -2.67 & $6.34 \times 10^{-62}$ & -2.56 & $6.33 \times 10^{-6}$ & -3.04 & $4.67 \times 10^{-24}$ \\
\hline NAT2 & -7.64 & $3.23 \times 10^{-18}$ & -5.62 & $2.58 \times 10^{-29}$ & -13.99 & $7.61 \times 10^{-107}$ & -36.89 & $4.54 \times 10^{-16}$ & -8.02 & $2.07 \times 10^{-34}$ \\
\hline SLC22A1 & -5.29 & $9.86 \times 10^{-11}$ & -6.2 & $1.56 \times 10^{-25}$ & -16.90 & $9.61 \times 10^{-65}$ & -15.41 & $1.23 \times 10^{-8}$ & -11.38 & $1.94 \times 10-21$ \\
\hline SLCO1B1 & -2.16 & $7.08 \times 10^{-05}$ & -3.63 & $3.50 \times 10^{-13}$ & -2.88 & $8.83 \times 10^{-33}$ & & & & \\
\hline SLCO1B3 & -4.16 & $4.37 \times 10^{-5}$ & -8.29 & $1.89 \times 10^{-18}$ & -11.48 & $2.97 \times 10^{-57}$ & -16.97 & $2.04 \times 10^{-9}$ & & \\
\hline UGT2B7 & -3.61 & $1.94 \times 10^{-12}$ & -3.64 & $5.98 \times 10^{-20}$ & & & & & -3.35 & $7.99 \times 10^{-21}$ \\
\hline
\end{tabular}

$\mathrm{N}$, normal; T, Tumor.

agreement with these findings, the downregulation of SULT1E1 and SULT2A1 in HCC was recently reported (Xie et al., 2017).

There are 21 functional $U G T$ genes in the human genome that are subdivided into four families: UGT1, UGT2, UGT3, and UGT8 ( $\mathrm{Hu}$ et al., 2014a). Eighteen UGT genes are classified as ADME genes, including four core ADME genes (1A1, 2B15, 2B17, and 2B7) and 14 extended ADME genes (1A3-10, 2A1, 2B4, 2B10, 2B11, 2B28, and UGT8) (www. pharmaadme.org). Of the ADME UGT genes, 12 (1A1, 1A3, $1 A 4,1 A 6,1 A 9,2 A 1,2 B 4,2 B 7,2 B 10,2 B 11,2 B 15$, and 2B17) were expressed in the TCGA HCC specimens (Fig. 3C; Table 1). We show here for the first time, UGT2B10 downregulation and UGT2B11 upregulation in $\mathrm{HCC}$ (Fig. $3 \mathrm{C}$ ). We also show UGT2B7 downregulation in HCC, a finding that was recently reported by others (Lu et al., 2015; Yan et al., 2015a). Previous studies have also reported downregulation of three other UGTs (1A1, 1A4, and 1A9) in HCC (Lu et al., 2015; Yan et al., 2015a). There was no overall significant deregulation of these three UGT genes in the TCGA HCC specimens (Fig. 3C) but their mRNA levels were decreased in HCC compared with paired adjacent noncancerous liver tissues in nearly $50 \%$ of the patients (Supplemental Fig. 1). As expected, the mRNA levels of the three extrahepatic UGTs (1A7, 1A8, and 1A10) were extremely low in the TCGA HCC specimens (data not shown).

Deregulation of ADME Genes Coding for Drug Transporters in HCC. Seventy-seven of the 298 ADME genes encode for drug transporters (PharmaADME) that belong to two transporter superfamilies: ABC and SLC transporters. Of these ADME genes, 54 (70\%) were expressed in the TCGA HCC specimens, including nine genes (17\%) that were downregulated and seven genes (13\%) that were upregulated in HCC. The details of the deregulation of $A B C$ and $S L C$ genes are described subsequently.

The human genome contains 48 functional $A B C$ genes (Dean et al., 2001; Wlcek and Stieger, 2014), among which 26 genes are defined as ADME genes (PharmaADME). Of these ADME $A B C$ genes, three $(A B C A 4, A B C C 4$, and $A B C C 10)$ were upregulated and one (ABCC9) was downregulated in HCC tumor
(Fig. 4A). The expression of three core ADME genes $(A B C B 1$, $A B C C 2$, and $A B C G 2$ ) showed high interindividual variabilities in HCC cancerous and noncancerous liver tissues (Chou et al., 1997; Richart et al., 2002; Zollner et al., 2005), and the degregulation of these $A B C$ genes in HCC has also been reported (Moustafa et al., 2004; Zollner et al., 2005; Borel et al., 2012). Indeed, we observed similar high interindividual expression variabilities for these three genes in the TCGA HCC specimens with upregulation or downregulation in $\mathrm{HCC}$ relative to corresponding adjacent noncancerous liver tissue in nearly $30 \%$ of the patients (data not shown).

The human genome contains 395 SLC genes (Hediger et al., 2013), among which 51 genes are defined as ADME genes (PharmaADME). Of these ADME SLC genes, 34 were expressed in the TCGA HCC specimens, including four genes that were upregulated (SLC22A4, SLC22A11, SLC22A15, and $S L C O 2 A 1)$ and eight genes that were downregulated (SLC10A1, SLC22A1, SLC22A10, SLC7A8, SLCO1B1, SLCO1B3, SLCO2B1, and SLCO4C1) in HCC (Fig. 4B; Table 1). The downregulation of three SLC genes (SLC22A1, $S L C O 1 B 1$, and SLCO1B3) in TCGA HCC was consistent with recent reports (Vavricka et al., 2004; Vander Borght et al., 2005; Tsuboyama et al., 2010; Heise et al., 2012). The downregulation of SLC22A3 in HCC was also reported (Heise et al., 2012). This gene was downregulated in nearly $50 \%$ of the TCGA HCC specimens. Northern blot analysis showed that $S L C O 4 C 1$ is only expressed in kidney, and thus is considered a kidney-specific SLC (Mikkaichi et al., 2004). We showed here that this gene was abundantly expressed in noncancerous liver tissues but was significantly downregulated in HCC (Fig. 4B).

Deregulation of ADME Genes Coding for Modifiers in HCC. Twenty-four of the 298 ADME genes encode for modifiers that either modulate the expression of ADME genes or affect the biochemistry of ADME enzymes (PharmaADME). Of these ADME modifiers, 21 were expressed in TCGA HCC specimens, including nine transcription factors (AHR, $A R N T$, HNF4A, PXR, CAR, PPARA, PPARD, PPARG, and RXRA) (Fig. 5; Table 1). Among these expressed modifier genes, four (catalase, cytidine deaminase, MAT1A, and PXR) were downregulated in HCC and one (KCNJ11) was upregulated in HCC. 

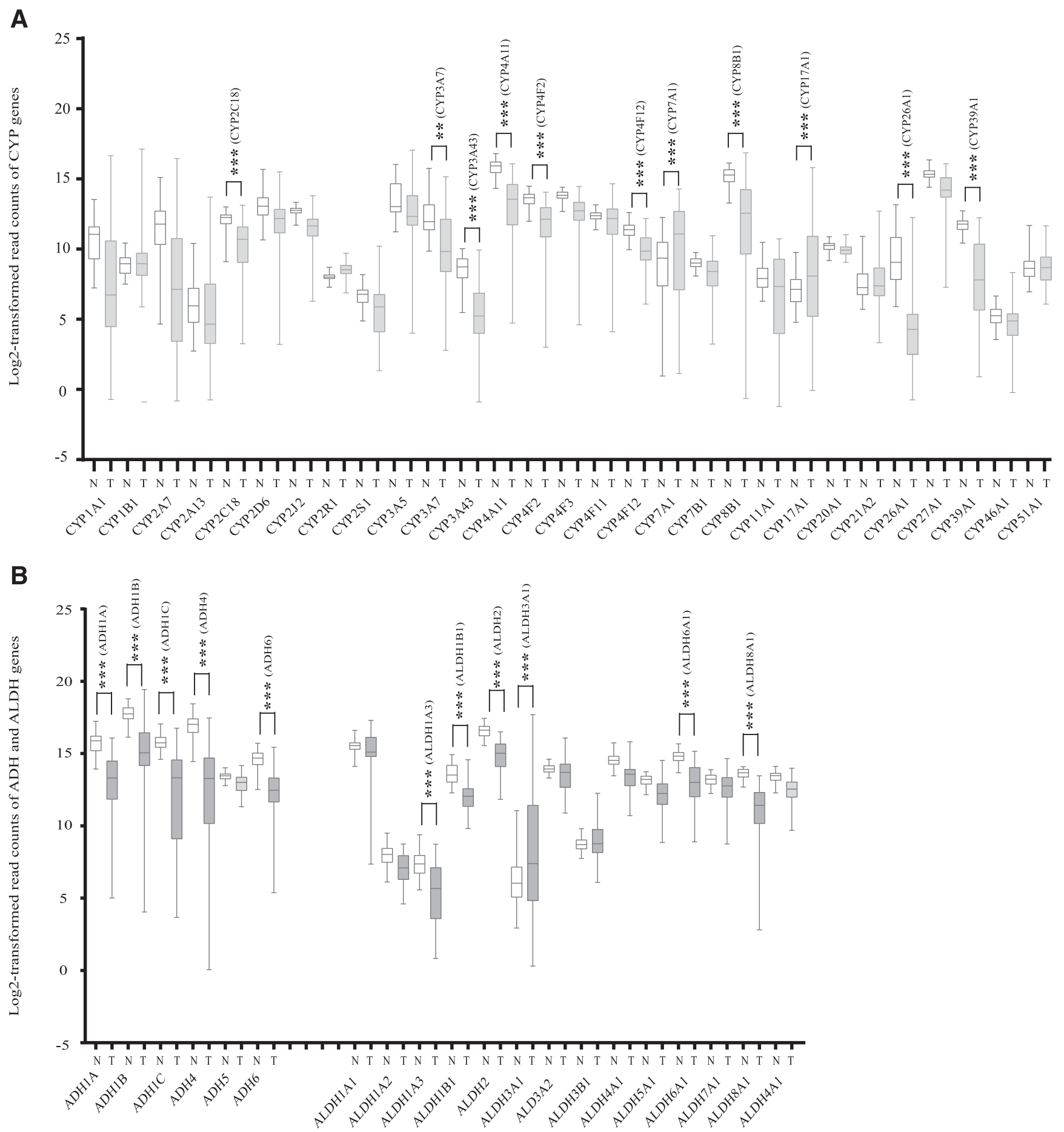

Fig. 2. Deregulation of phase I drug metabolizing enzymes in HCC. The TCGA LIHC RNA-seq data set representing 50 paired HCC cancerous and corresponding adjacent noncancerous liver tissues was downloaded from the Genomic Data Commons Data Portal (https://portal.gdc.cancer.gov/) and subjected to differential gene expression analysis using the DESeq2 program as described in Materials and Methods. The box-and-whisker plot shows the log 2-transformed expression levels (minimum, first quartile, median, third quartile, and maximum) of $29 \mathrm{ADME}$ genes (A) and six ADH and $14 \mathrm{ALDH}$ genes (B) in $50 \mathrm{HCC}$ cancer tissues and their corresponding adjacent noncancerous liver tissues. ${ }^{*} P<0.05$; $* * * P<0.01$; $* * * P<0.001$. CYP, cytochrome P450. N, noncancerous liver tissues; T, HCC cancerous liver tissues.

The upregulation of KCNJ11 (Zhang et al., 2018) and the downregulation of catalase (Cho et al., 2014), PXR (Chen et al., 2014), or cytidine deaminase (Nwosu et al., 2017) in HCC have been recently reported. KCNJ11 is an HCC oncogene and its overexpression in HCC promotes cell proliferation and tumor progression (Zhang et al., 2018). MAT1A upregulation in HCC was also recently reported (Nwosu et al., 2017); however, the expression of this gene was significantly downregulated in the TCGA HCC specimens. This discrepancy awaits further investigation.

In addition to the nine transcription factors defined as ADME modifiers by the PharmaADME Consortium, many 


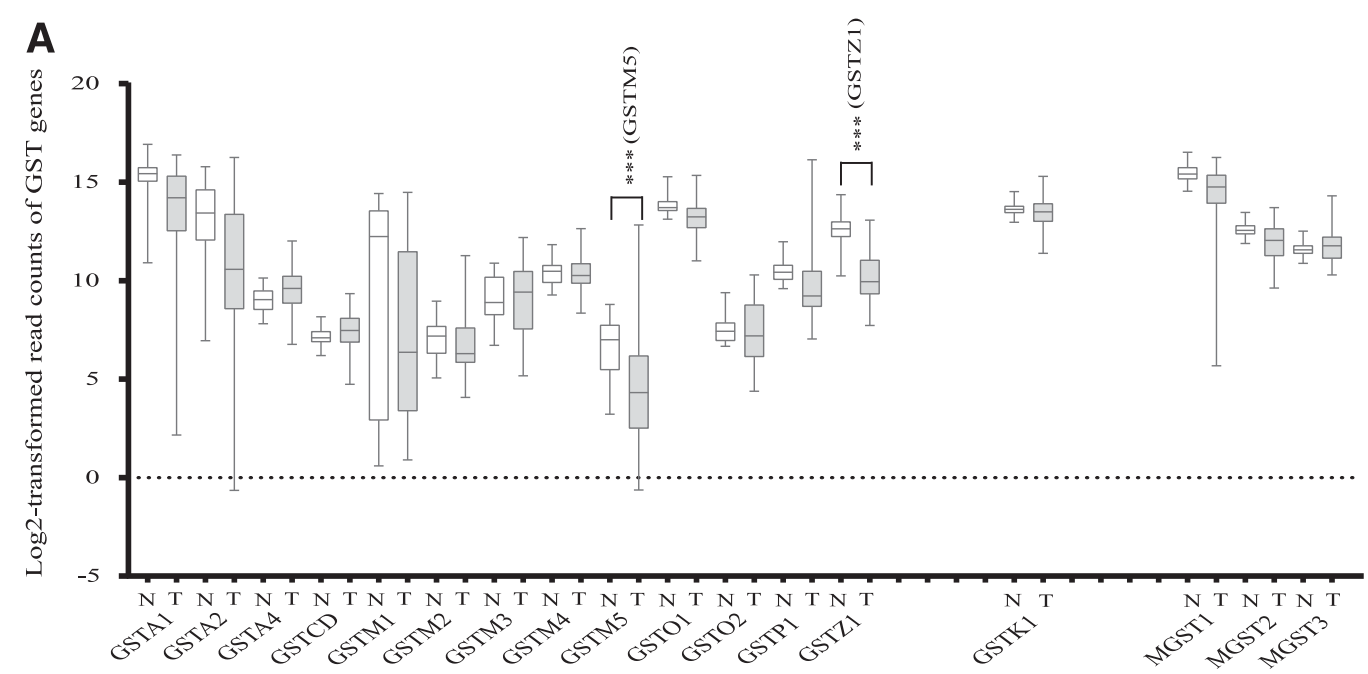

Cytosolic GSTs

Mitochondrial GST Microsomal GSTs

B

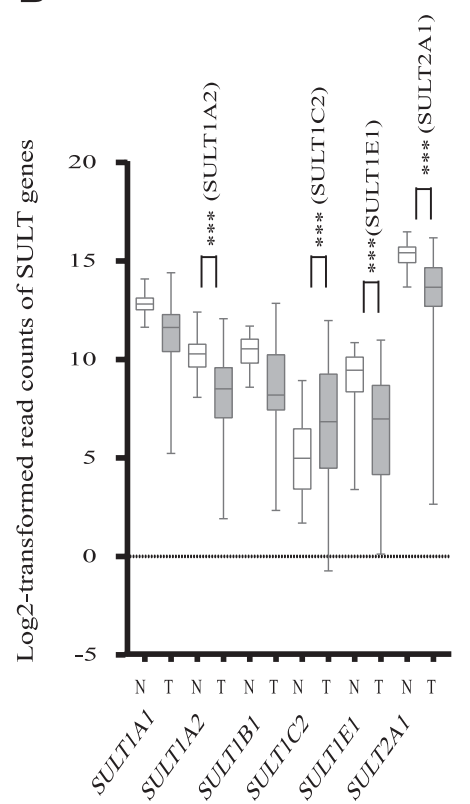

C

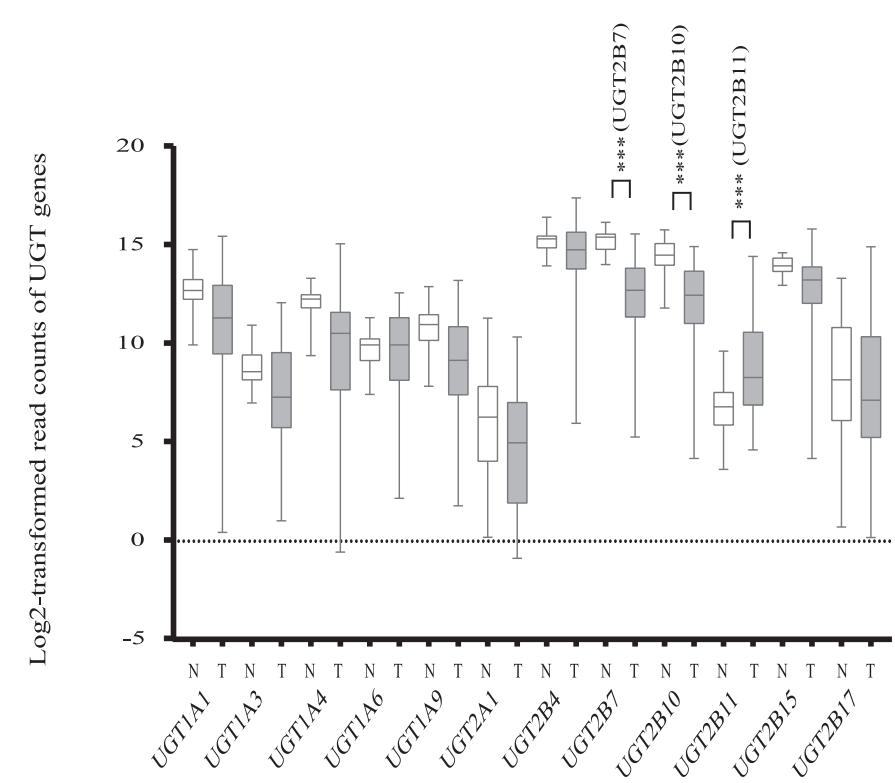

Fig. 3. Deregulation of phase II drug metabolizing enzymes in HCC. The TCGA LIHC RNA-seq data set representing 50 paired HCC cancerous and corresponding adjacent noncancerous liver tissues was downloaded from the Genomic Data Commons Data Portal (https://portal.gdc.cancer.gov/) and subjected to differential gene expression analysis using the DESeq2 program as described in Materials and Methods. The box-and-whisker plot shows the distribution (minimum, first quartile, median, third quartile, and maximum) of the log 2-transformed expression levels of (A) 17 GST genes of three subfamilies (cytosolic, mitochondrial, and microsomal), (B) six SULT genes, and (C) 12 UGT genes in 50 HCC cancer tissues and their corresponding adjacent noncancerous liver tissues. ${ }^{* * *} P<0.001$. N, noncancerous liver tissues; $\mathrm{T}, \mathrm{HCC}$ cancerous liver tissues.

other transcription factors are involved in transcription regulation of ADME genes (Hu et al., 2014a; Zanger and Schwab, 2013). For example, a large number of ADME genes are regulated by the oxidative stress-responsive transcription factor Nrf2 (Hayes and Dinkova-Kostova, 2014). Mutations of $N R F 2$ and its negative regulator KEAP1 in HCC have been reported (Guichard et al., 2012). There was no significant overall deregulation of NRF2 and KEAP1 in TCGA HCC specimens (Fig. 5); however, we show here that NRF2 had reduced mRNA levels in HCC in 42 of the 50 patients (Supplemental Fig. 2).

Correlation Analyses Between Core ADME Genes and Nine ADME Modifiers Coding for Transcription Factor Genes in HCC. As mentioned previously, nine
ADME modifiers (AHR, ARNT, HNF4A, PXR, CAR, PPARA, $P P A R D, P P A R G$, and $R X R A$ ) are transcription factors that are believed to be able to alter the expression of other ADME genes. Indeed, three of these modifiers (PXR, CAR, and HNF4A) are involved in transcriptional regulation of some ADME genes in liver (Xu et al., 2005; Congiu et al., 2009; Zhong et al., 2017). Analysis of TCGA RNA-seq data from $371 \mathrm{HCC}$ tumor tissues revealed that most of the core ADME genes were significantly positively correlated to five TF modifiers (HNF4A, PXR, CAR, PPARA, and RXRA), and that about one-half of the core ADME genes were significantly positively correlated to two other TF modifiers (AHR and $A R N T$ ) (Supplemental Table 14). In particular, PXR was significantly positively correlated with 25 core ADME genes 


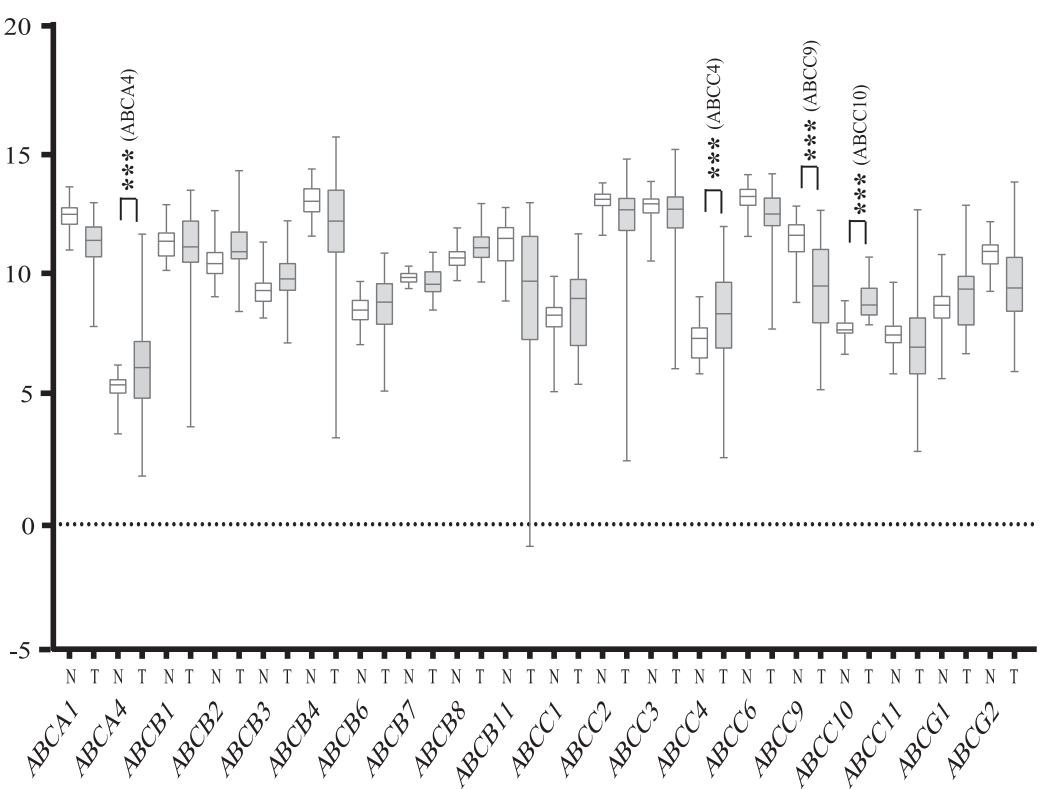

B

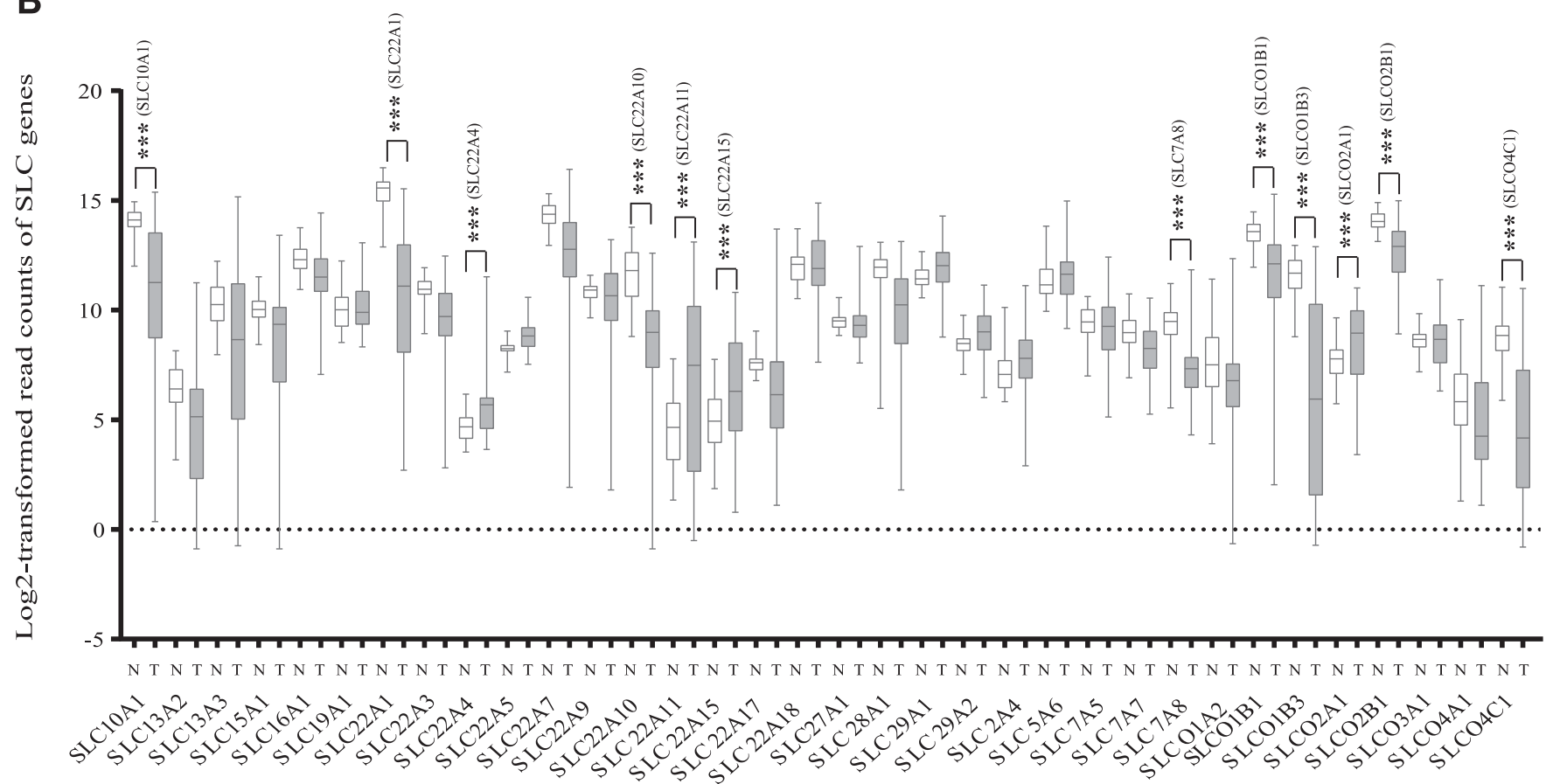

Fig. 4. Deregulation of drug transporter genes in HCC. The TCGA LIHC RNA-seq data set representing 50 paired HCC cancerous and corresponding adjacent noncancerous liver tissues was downloaded from the Genomic Data Commons Data Portal (https://portal.gdc.cancer.gov/) and subjected to differential gene expression analysis using the DESeq2 program as described in Materials and Methods. The box-and-whisker plot shows the distribution (minimum, first quartile, median, third quartile, and maximum) of the log 2-transformed expression levels of (A) $20 A B C$ transporter genes and (B) $37 S L C$ transporter genes in $50 \mathrm{HCC}$ cancer tissues and their corresponding adjacent noncancerous liver tissues. ${ }^{* * *} P<0.001$. N, noncancerous liver tissues; T, HCC cancerous liver tissues.

(Fig. 6). By contrast, PPARD was only positively correlated with one core ADME gene (DPYD) but was negatively correlated to 17 core ADME genes (Fig. 7). Similarly, PPARG was positively correlated with only four core ADME genes (ABCC2, CYP2E1, SLCO1B3, and UGT1A1) but negatively correlated with 14 core ADME genes (Supplemental Table 14). Of note, GSTP1 was the only core ADME gene that was significantly negatively correlated with most of the modifier TFs (seven out of nine) (Fig. 8). We further examined whether these TFs were differentially expressed in HCC in individual patients relative to their paired adjacent noncancerous liver tissues. As expected from an overall downregulation of $P X R$ in HCC (Fig. 5), it was downregulated in HCC in almost all patients (Supplemental Fig. 2). There was no significant overall deregulation of $A H R, C A R, P P A R D$, and PPARG (Fig. 5), but we show here that $A H R$ and $C A R$ were downregulated in HCC in nearly $50 \%$ of the patients, and that there was upregulation of PPARD and PPARG in HCC in nearly 


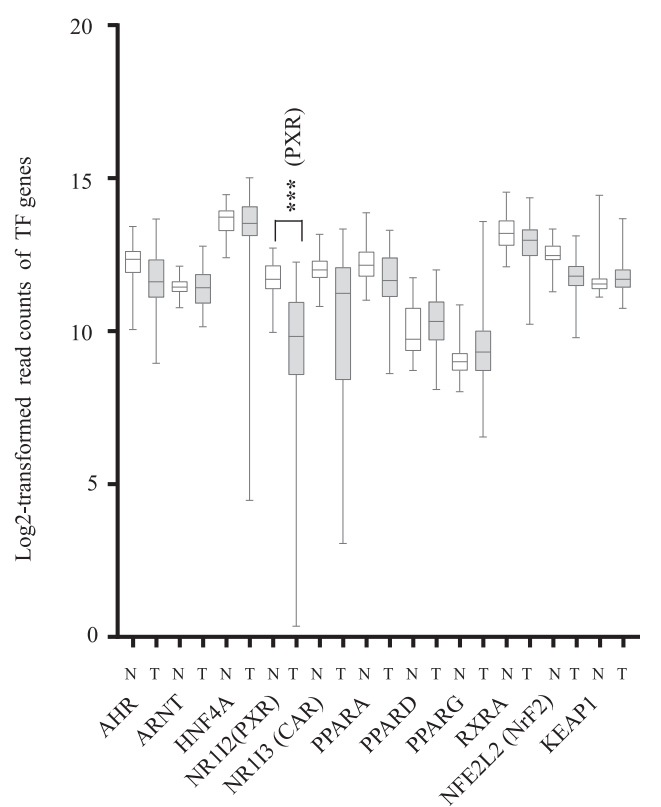

Fig. 5. Deregulation of genes coding for nine ADME modifier transcription factors and NRF2 and its negative regulator KEAP1 in HCC. The TCGA LIHC RNA-seq data set representing 50 paired HCC cancerous and corresponding adjacent noncancerous liver tissues was downloaded from the Genomic Data Commons Data Portal (https://portal.gdc.cancer.gov/) and subjected to differential gene expression analysis using the DESeq2 program as described in Materials and Methods. The box-and-whisker plot shows the distribution (minimum, first quartile, median, third quartile, and maximum) of the $\log 2$-transformed expression levels of nine transcription factors that are classified as ADME modifiers. ${ }^{* * *} P<0.001$. $\mathrm{N}$, noncancerous liver tissues; T, HCC cancerous liver tissues.

$50 \%$ of the patients (Supplemental Fig. 2). Taken together, about one-half of core ADME genes are positively correlated to seven ADME TFs (AHR, ARNT, HNF4A, PXR, CAR, PPARA, and $R X R A$ ) and negatively correlated to two ADME TFs (PPARD and PPARG) in HCC. Furthermore, NRF2 was also positively correlated with more than 20 core ADME genes (Supplemental Table 14). Given that six of these 11 transcription factors and their coregulators are deregulated in HCC, it is likely that they contribute to the widespread downregulation of core ADME genes in HCC.

The Expression Levels of Core ADME Genes Are Correlated with Each Other in HCC. The correlation of the core ADME genes with ADME TFs described previously suggested that core ADME genes are coregulated. To examine this idea, we performed expression correlation analysis of all 28 core $\mathrm{ADME}$ genes. Twenty-two core ADME genes $(A B C B 1$, $A B C C 2, A B C G 2, C Y P 1 A 1, C Y P 1 A 2, C Y P 2 A 6, C Y P 2 B 6$, CYP2C8, CYP2C9, CYP2D6, CYP3A4, CYP3A5, NAT1, NAT2, SLC22A1, SLCO1B1, SULT1A1, TPMT, UGT1A1, UGT2B7, UGT2B15, and UGT2B17) were significantly positively correlated with at least 20 other core ADME genes (Supplemental Table 15); five core ADME genes (CYP2C19, CYP2E1, DPYD, GSTM1, and SLCO1B3) were significantly positively correlated with about one-half of the core ADME genes (Supplemental Table 15). For instance, UGT2B7 was significantly positively correlated with 25 core ADME genes (Fig. 9). GSTP1 was the only core ADME gene that was significantly negatively correlated to more than one-half of the core ADME genes (Supplemental Table 15), consistent with the aforementioned negative correlation of GSTP1 with seven
ADME TFs. Taken together, the widespread expression correlation of core ADME genes with each other and with transcription factors as summarized in Supplemental Table 16 support the hypothesis that $\mathrm{ADME}$ genes are coordinately regulated by transcription factors in the liver.

Deregulation of miRNAs that Are Known to Regulate Core ADME Genes in HCC. miRNAs generally repress gene expression through mRNA degradation or translational repression. Therefore, the upregulation of miRNAs that regulates $\mathrm{ADME}$ genes might contribute to their downregulation in HCC. To test this hypothesis, we examined the expression levels of 12 miRNAs (miR-132, miR-128, miR-130b, miR-29a-3p, miR-27a, miR-107, miR-25, miR-629, miR-1035p, miR-378a, miR-126, and miR-142) (Supplemental Table 4) that are reported to target the downregulated core ADME genes (CYP1A2, CYP2C9, CYP2C9, CYP2C19, CYP3A4, CYP2C8, CYP2B6, SLCO1B3, CYP2C8, CYP2E1, CYP2A6, and $U G T 2 B 7$, respectively) in $\mathrm{HCC}$ and paired adjacent noncancerous liver tissues (Mohri et al., 2010; Zhang et al., 2012; Nakano et al., 2015; Rieger et al., 2015; Shi et al., 2015; Yu et al., 2015a,b; Higuchi et al., 2016; Jin et al., 2016; Chen et al., 2017; Papageorgiou and Court, 2017). Strikingly, most (eight out of 12) of these miRNAs were upregulated in HCC tumors (Fig. 10), suggesting that they may play an important role in core ADME gene dysregulation in HCC.

Deregulation of Aldo-Keto Reductases in HCC. Aldoketo reductases (AKRs) are involved in the phase I metabolism of numerous endogenous and xenobiotic compounds including steroids, carbohydrates, prostaglandins, aldehydes, ketones, and many therapeutic drugs such as the cytotoxic anthracyclines (e.g., doxorubicin, daunorubicin, and idarubicin) (Novotna et al., 2008; Skarka et al., 2011; Matsunaga et al., 2012; Penning, 2015). There are $15 A K R$ genes in the human genome (Penning, 2015) but none of these genes are classified as ADME genes by the PharmaADME consortium (pharmadame.org). We show here that 11 human $A K R$ genes (e.g., $A K R 1 A 1, A K R 1 B 1, A K R 1 B 10$, AKR1C1, AKR1C2, AKR1C3, AKR1C4, AKR1D1, AKR1E2, $A K R 7 A 2$, and $A K R 7 A 3)$ were expressed in TCGA HCC specimens (Fig. 11). Of these genes, $A K R 1 B 10$ and $A K R 1 C 3$ were significantly upregulated, whereas $A K R 1 D 1$ and $A K R 7 A 3$ were significantly downregulated in HCC. $A K R 1 B 10$ upregulation is consistent with the overexpression of AKR1B10 enzyme in HCC as reported recently in an immunohistochemistry study (Matkowskyj et al., 2014). AKR7A3 downregulation in HCC (Chow et al., 2016) and AKR1C3 upregulation in prostate and breast cancer (Lin et al., 2004; Fung et al., 2006) has also been reported.

\section{Discussion}

ADME genes are involved in drug absorption, distribution, metabolism, and excretion and are abundantly expressed in the liver, the major organ of drug metabolism and clearance in humans. Although several studies have investigated the deregulation of subsets of ADME genes in HCC (Okabe et al., 2001; Lee et al., 2004; Park et al., 2006; Borel et al., 2012; Chen et al., 2014; Ho et al., 2015; Jin et al., 2015a; Yan et al., 2015a,b; Woo et al., 2017), there has been no systematic, targeted analysis of the entire ADME gene set. In this study, our comprehensive analysis of paired cancerous and noncancerous specimens from the TCGA HCC data set identified 

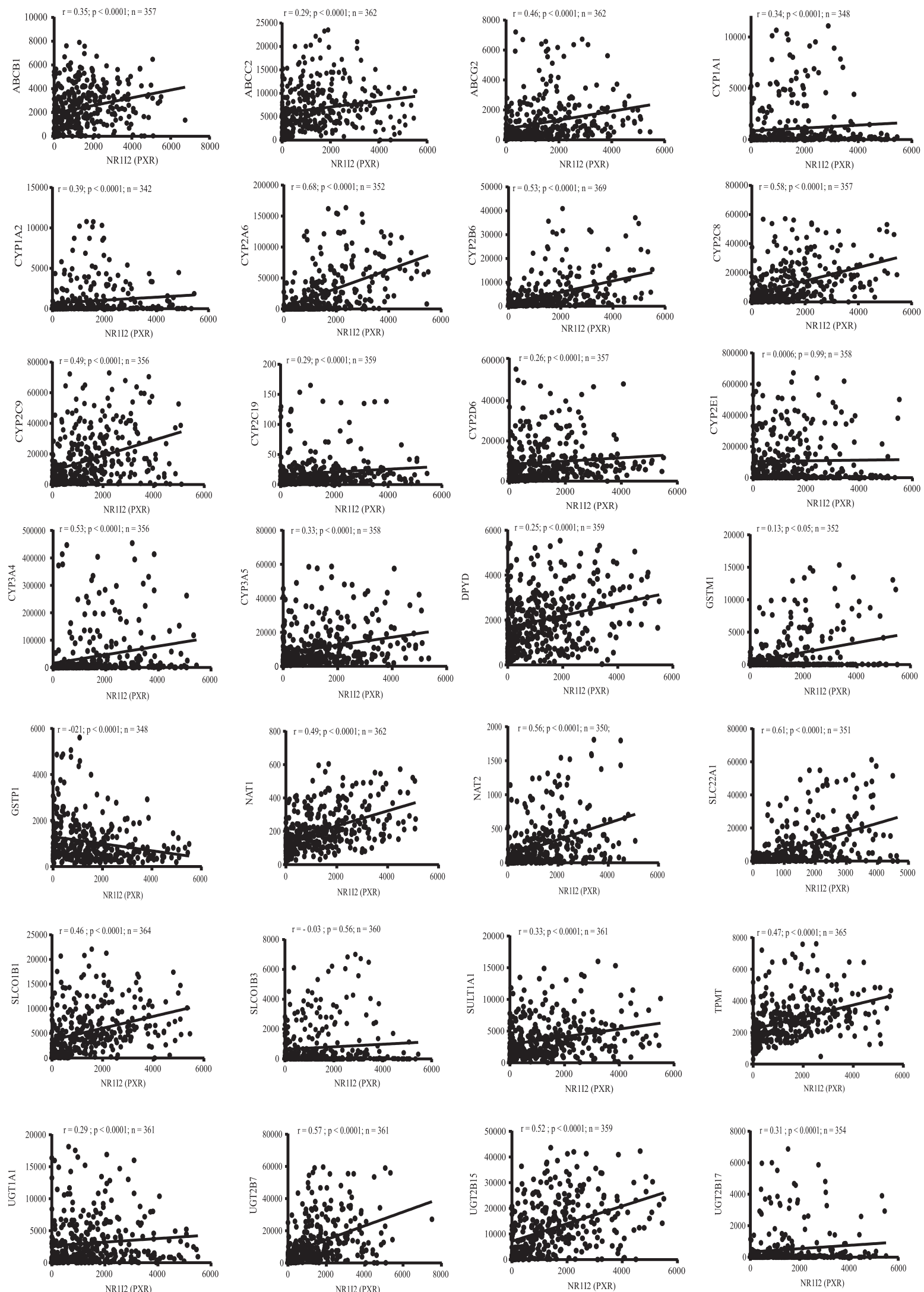

Fig. 6. Correlation analysis between PXR and core ADME genes in HCC. The TCGA LIHC RNA-seq data set representing 371 HCC tumors was downloaded from the Genomic Data Commons Data Portal (https://portal.gdc.cancer.gov/) and subjected to Spearman's ranking correlation analysis using GraphPad Prism version 7.03. The graphs shown are the correlation analyses of the expression (mRNA) levels between PXR and 28 core ADME genes. A $P$ value of $<0.05$ was considered statistically significant. $r$, correlation coefficient. 

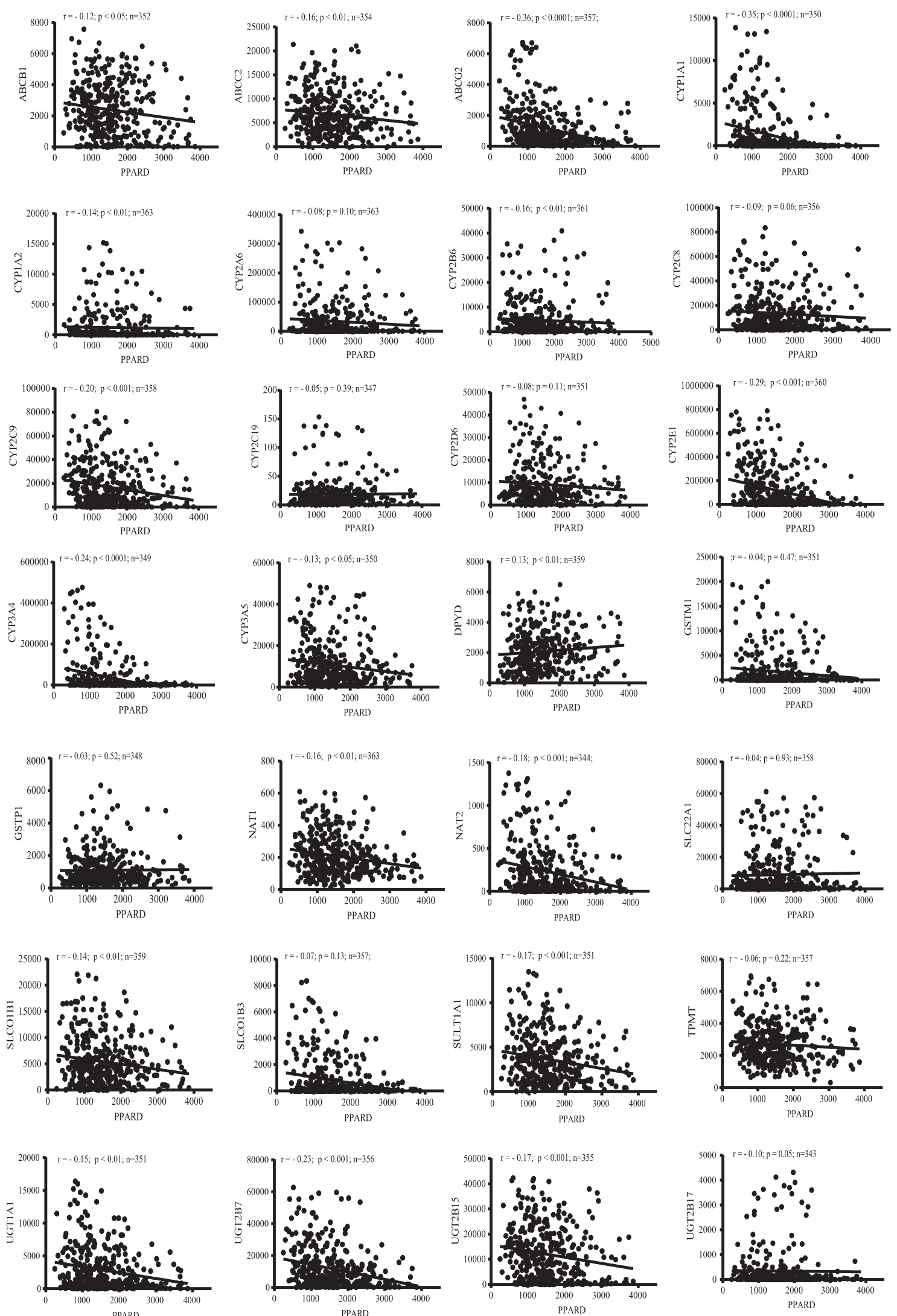

Fig. 7. Correlation analysis between PPARD and core ADME genes in HCC. The TCGA LIHC RNA-seq data set representing 371 HCC tumors was downloaded from the Genomic Data Commons Data Portal (https://portal.gdc.cancer.gov/) and subjected to Spearman's ranking correlation analysis using GraphPad Prism version 7.03. The graphs shown are the correlation analyses of the expression (mRNA) levels between PPARD and 28 core ADME genes. A $P$ value of $<0.05$ was considered statistically significant. $r$, correlation coefficient. 

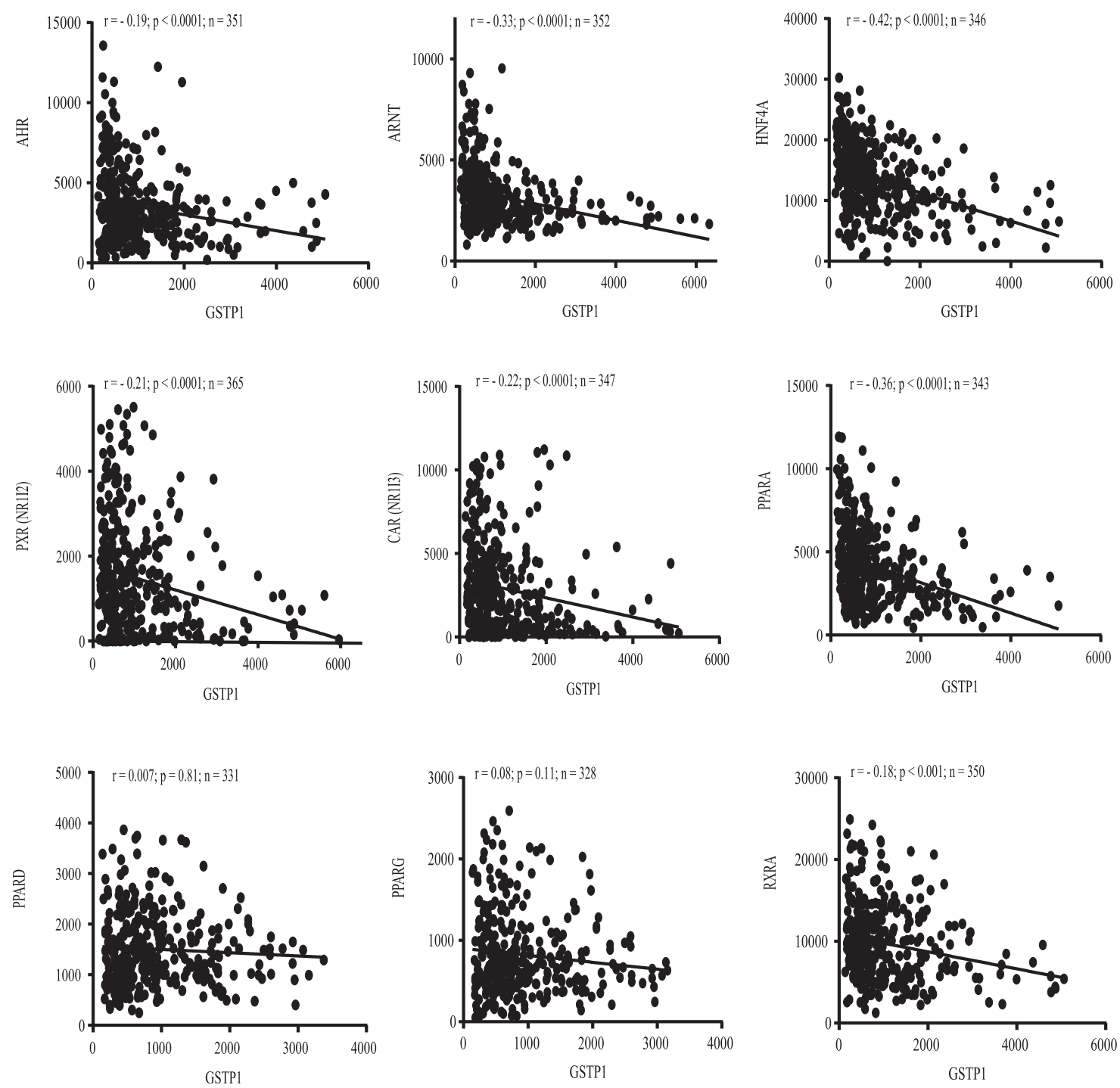

Fig. 8. Correlation analysis between GSP1 and nine transcription factors that are classified as ADME modifiers in HCC. The TCGA LIHC RNA-seq data set representing $371 \mathrm{HCC}$ tumors was downloaded from the Genomic Data Commons Data Portal (https://portal.gdc.cancer.gov/) and subjected to Spearman's ranking correlation analysis using GraphPad Prism version 7.03. The graphs shown are the correlation analyses of the expression (mRNA) levels between GSP1 and nine TFs that are classified as ADME modifiers. A $P$ value of $<0.05$ was considered statistically significant. $\mathrm{r}$, correlation coefficient.

for the first time the complete list of $\mathrm{ADME}$ genes that are deregulated in HCC. Overall, around one-quarter of all ADME genes were downregulated while only $6 \%$ were upregulated in HCC. The deregulated genes included members of all four groups of ADME genes, namely, phase I (CYPs, $A D H s$, and $A L D H s)$ and phase II (GSTs, SULTs, and UGTs) drug metabolizing enzymes, drug transporters ( $A B C$ s and $S L C \mathrm{~s}$ ), and modifiers. Moreover, one-half of the core ADME genes were significantly downregulated in HCC. Among the ADME gene superfamilies, CYPs were the most significantly downregulated. Our analyses of four other microarray gene expression profiling reports of $\mathrm{HCC}$ observed similar downregulation of these core ADME genes in HCC (Chen et al., 2002; Wurmbach et al., 2007; Roessler et al., 2010; Thurnherr et al., 2016). The deregulation of many other ADME genes reported by us in the present study is consistent with the findings of previous studies as already discussed comprehensively. However, we report in the present study for the first time on the deregulation of a variety of ADME genes in HCC, such as AKR1C3, AKR1D1, ALDH1A3, ALDH3A1, ALDH6A1, $A L D H 8 A 1, U G T 2 B 10$, and UGT2B11. Most ADME genes belong to superfamilies that also contain non-ADME members (for example, genes that mediate biosynthetic processes or transport endobiotics). To better understand the specificity of ADME deregulation, we compared the ADME gene set to nonADME genes from the same superfamilies. Our results showed that the ADME genes were far more likely to be downregulated than non-ADME genes in HCC. We further performed unbiased pathway analysis (Reactome) (https:// reactome.org) using the entire $\mathrm{ADME}$ and non-ADME gene set (609 genes) and found that the downregulated gene set was significantly more enriched for drug metabolism pathways than the unchanged or upregulated gene sets (data not shown). Together with the data on ADME TFs and miRNAs discussed subsequently, this supports the idea that ADMEspecific regulatory pathways are deregulated in HCC. 

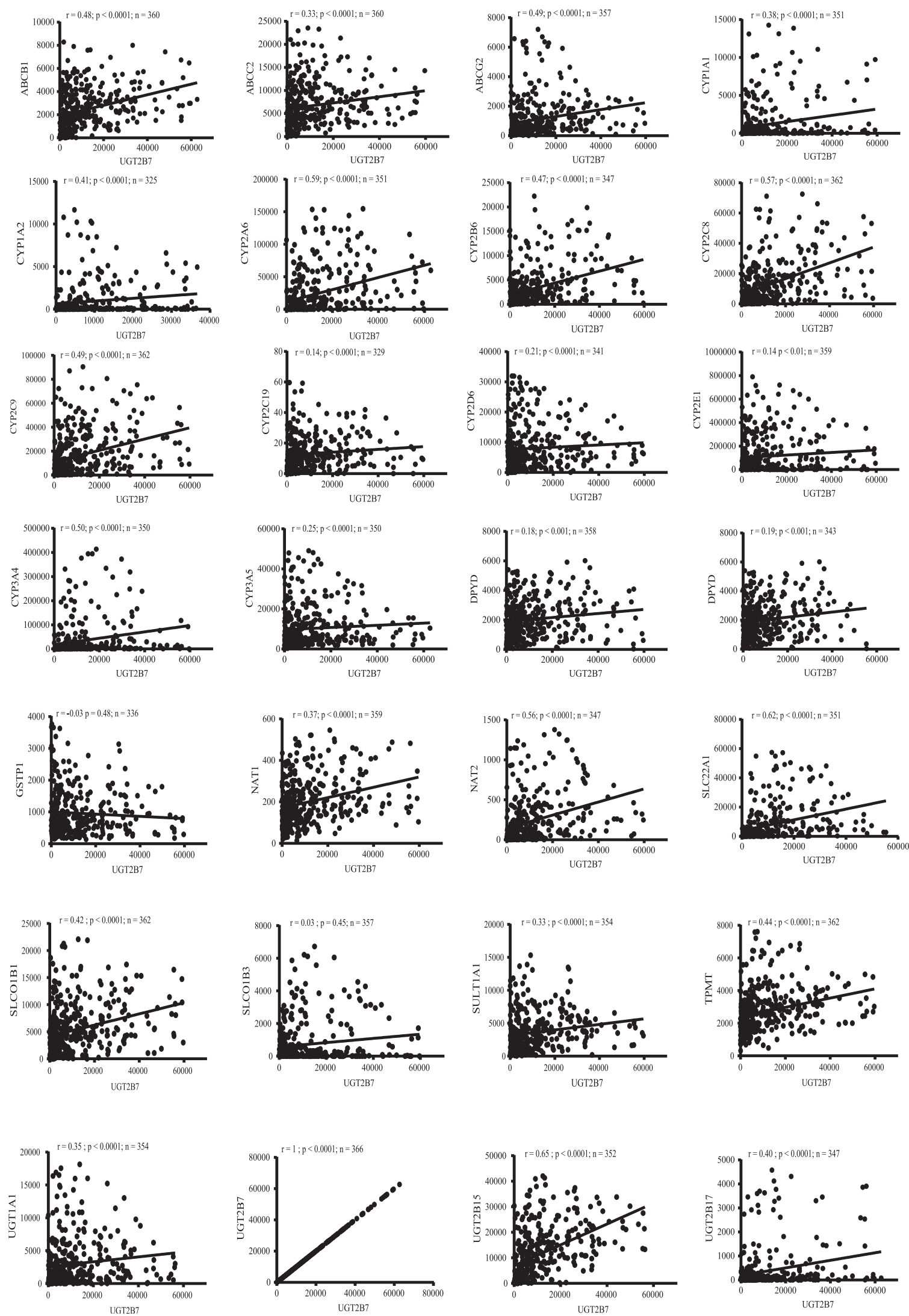

Fig. 9. Correlation analysis of the expression levels between UGT2B7 and 27 other core ADME genes in HCC. The TCGA LIHC RNA-seg data set representing $371 \mathrm{HCC}$ tumors was downloaded from the Genomic Data Commons Data Portal (https://portal.gdc.cancer.gov/) and subjected to Spearman's ranking correlation analysis using GraphPad Prism version 7.03. The graphs shown are the correlation analyses of the expression (mRNA) levels between UGT2B7 and 27 other core ADME genes. A $P$ value of $<0.05$ was considered statistically significant. $r$, correlation coefficient. 

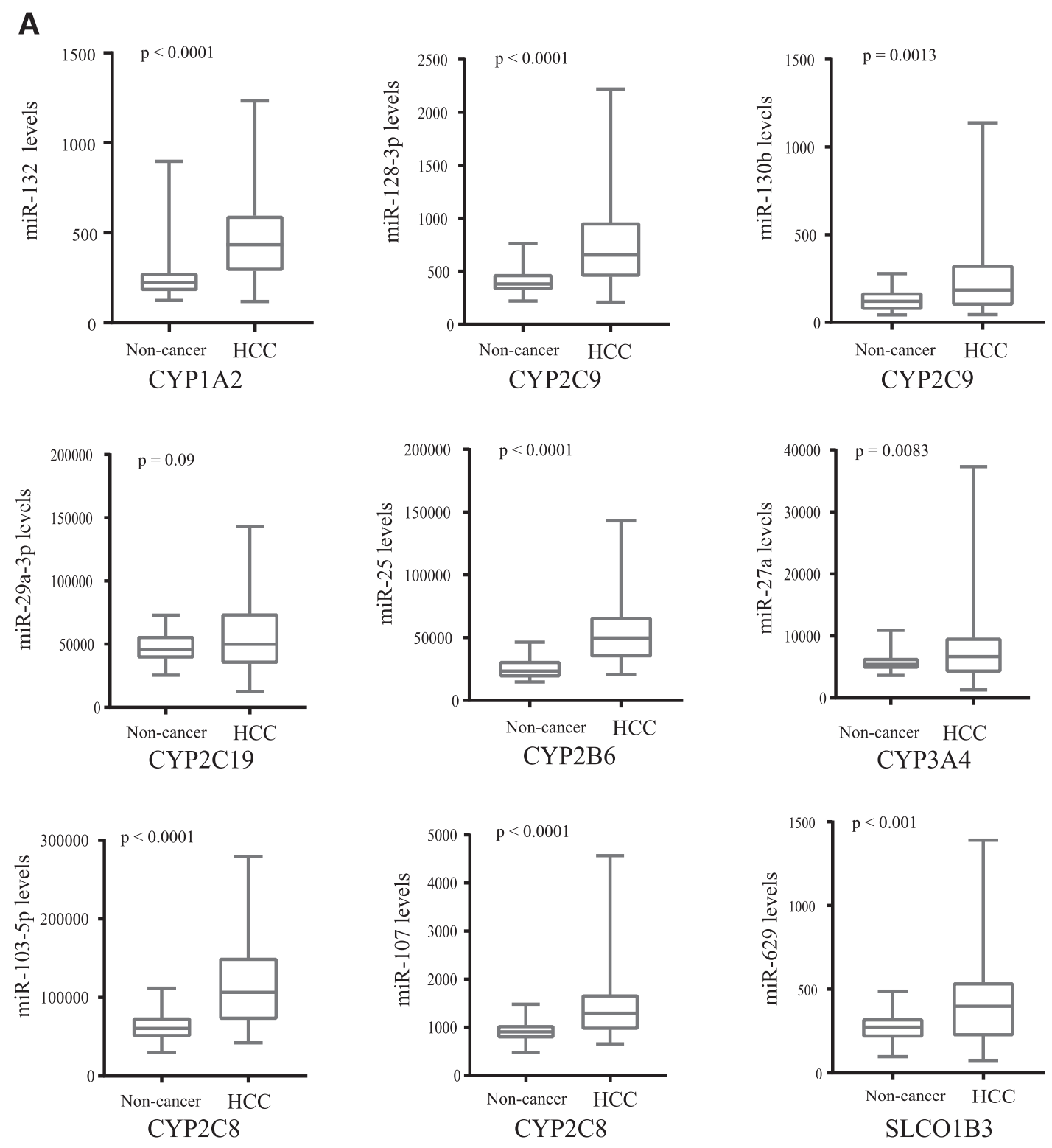

B
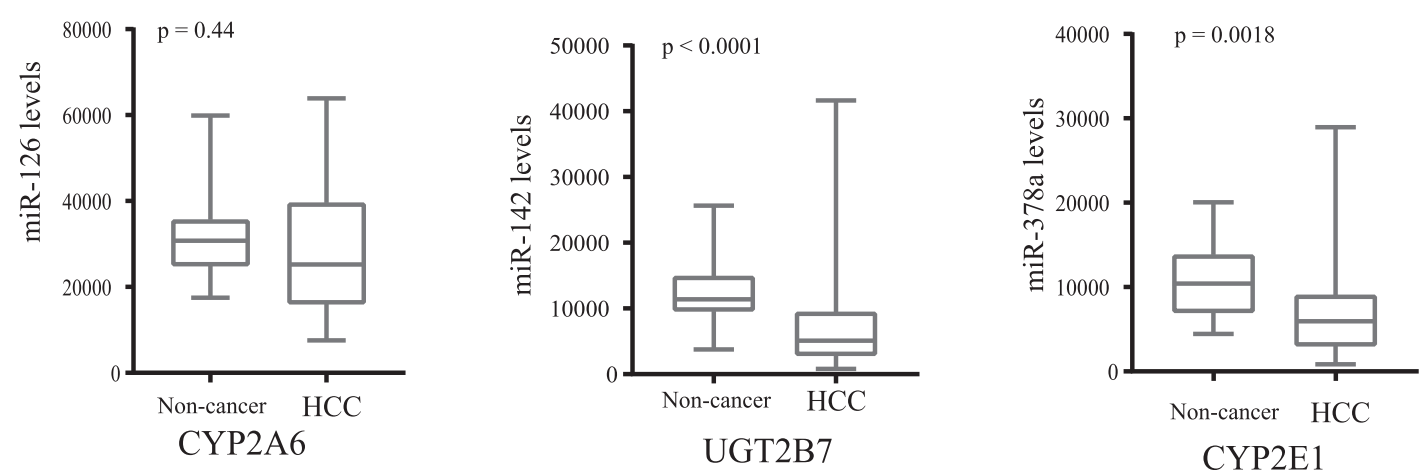

Fig. 10. Deregulation of miRNAs in HCC. The TCGA LIHC miRNA-seq data set representing 49 paired HCC and corresponding adjacent noncancerous liver tissues was downloaded from The Cancer Genome Atlas Data Portal (https://gdc-portal.nci.nih.gov/) and normalized using the upper quantile normalization method. The differences in the expression levels of 12 miRNAs between HCC cancerous and corresponding adjacent normal noncancerous liver tissues were statistically assessed using GraphPad Prism (version 7.03) ( $t$ test). A $P$ value of $<0.05$ was considered statistically significant. 


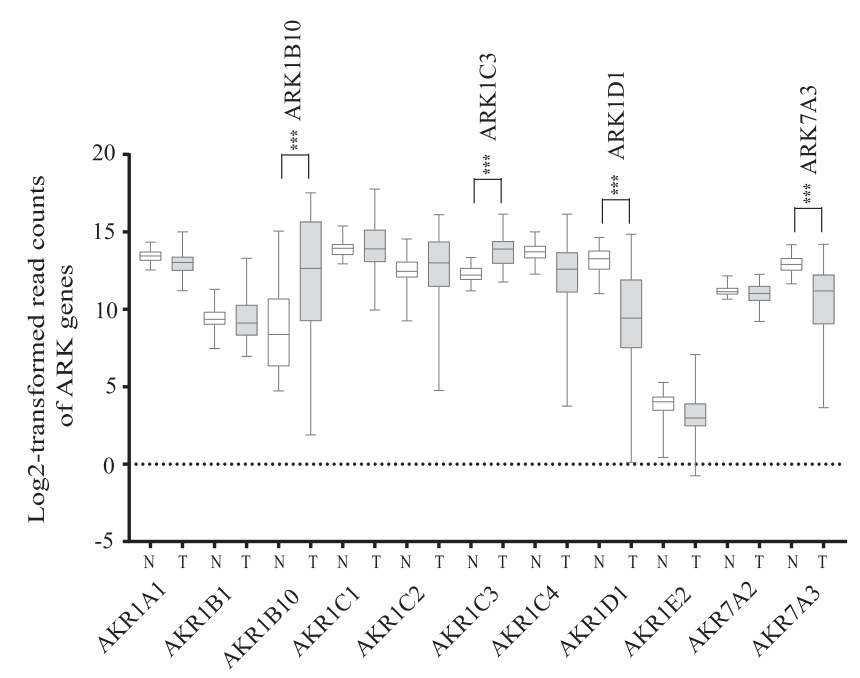

Fig. 11. Deregulation of AKRs in HCC. The TCGA LIHC RNA-seq data set representing 50 paired $\mathrm{HCC}$ cancerous and corresponding adjacent noncancerous liver tissues was downloaded from the Genomic Data Commons Data Portal (https://portal.gdc.cancer.gov/) and subjected to differential gene expression analysis using the DESeq2 program as described in Materials and Methods. The box-and-whisker plot shows the distribution (minimum, first quartile, median, third quartile, and maximum) of the log 2-transformed expression levels of 11 human AKR genes in $50 \mathrm{HCC}$ cancer tissues and their corresponding adjacent noncancerous liver tissues. $* * * P<0.001$. N, noncancerous liver tissues; $\mathrm{T}, \mathrm{HCC}$ cancerous liver tissues.

Decreased expression of genes encoding hepatocyte-specific gene products (e.g., albumin) and detoxification enzymes (CYPs) and associated loss of liver-specific functions are recognized phenomena in HCC and are in part due to de-differentiation of cancer cells (Okabe et al., 2001; Park et al., 2006; Andrisani et al., 2011; Obaidat et al., 2012). The widespread downregulation of ADME genes in HCC shown herein is likely part of this de-differentiation. The molecular mechanisms underlying de-differentiation are not completely understood but could be related to epigenetic (chromatin) modifications or deregulation of transcription factors and miRNAs that regulate liver-specific genes. The deregulation of TFs and miRNAs in HCC, as we showed herein in this study, supports this assumption. Briefly, we found that most of the miRNAs known to regulate the downregulated core ADME genes were upregulated in HCC. Furthermore, transcription factors $(P X R, A H R$, and $C A R)$ that are positively correlated with core ADME genes were downregulated in HCC, whereas the transcription factors (PPARD and $P P A R G)$ that are negatively correlated with core ADME genes were upregulated. The recent reports of DNA hypermethylation at the promoters of three downregulated core ADME genes (CYP1A2, CYP2C9, and SLC22A1) in HCC links epigenetic modifications to their deregulation in this cancer (Schaeffeler et al., 2011; Shen et al., 2012).

There are no curative therapeutic options for advanced HCC (Llovet et al., 2008). Most of the clinical trials evaluating anticancer agents in chemotherapy, hormone therapies, and immunotherapy have failed to show convincing survival benefits for patients with advanced HCC (Llovet et al., 2008; Llovet and Hernandez-Gea, 2014). The failure of these trials might be related to the expression of nearly $80 \%$ of $\mathrm{ADME}$ genes (233 out of 298) in HCC as we found in the present study. Two multikinase inhibitors, sorafenib and regorafenib, are currently the only two drugs approved by the Food and Drug Administration for treating advanced HCC (Llovet et al., 2008; Bruix et al., 2017). The deregulation of the ADME genes involved in the metabolism and clearance of these two drugs may help to explain their relative efficacy. The key ADME genes involved in the metabolism and clearance of sorafenib and regorafenib in liver include the enzymes CYP3A4 (Ghassabian et al., 2012) and UGT1A9 (Peer et al., 2012; Miners et al., 2017), influx transporters (SLCO1B1, SLCO1B3, and SLC22A1) (Swift et al., 2013; Zimmerman et al., 2013; Ohya et al., 2015), and efflux transporters ( $A B C B 1, A B C C 2$, and $A B C G 2$ ) (van Erp et al., 2009; Ohya et al., 2015). While the efflux transporters were not significantly altered in HCC, the expressions of CYP3A4, UGT1A9, $S L C O 1 B 1, S L C O 1 B 3$, and $S L C 22 A 1$ were all significantly downregulated in HCC, consistent with previous observations (Vander Borght et al., 2005; Tsuboyama et al., 2010; Heise et al., 2012; Ye et al., 2014). The downregulation of CYP3A4 and UGT1A9 could reduce the metabolism of sorafenib and regorafenib, and hence enhance therapeutic efficacy; however, the downregulation of influx transporters (SLCO1B1, $S L C O 1 B 3$, and SLC22A1) may decrease the cellular uptake and reduce efficacy. Ultimately, the combined effects of deregulated uptake and metabolism will determine the therapeutic efficacy of sorafenib and regorafenib. The opposing influence of ADME gene deregulation on the therapeutic efficacy of this effective HCC therapeutic regimen warrants further investigation.

Our observation of deregulated UGT expression may also provide some insights into cytotoxic drug efficacy in HCC. The chemotherapy regimen epirubicin, 5-fluoruracil, and cisplatin has been found to be poorly effective as a first line therapy for HCC; however, it did provide survival benefits when used as a second line therapy for advanced sorafenib-resistant HCC (Lee et al., 2014). UGT2B7 is the only UGT known to mediate epirubicin conjugation (Innocenti et al., 2001), and this metabolism is suggested to be responsible for its lower systemic toxicity relative to doxorubicin (Hu et al., 2014b). We observed downregulation of UGT2B7 in HCC as well as upregulation of UGT2B11. In recent studies performed in vitro, we found that UGT2B11 inhibits the ability of UGT2B7 to glucuronidate epirubicin (Meech et al, manuscript in preparation). Thus, the combined downregulation of UGT2B7 and upregulation of UGT2B11 could reduce intratumoural epirubicin conjugation and clearance, and this may contribute to the modest efficacy of this regimen in advanced HCC.

CBR1 and multiple AKR subfamily 1 enzymes (AKR1A1, AKR1B1, AKR1B10, AKR1C3, and AKR1C4) are involved in metabolism of doxorubicin, a common cytotoxic drug for treating HCC (Kassner et al., 2008; Novotna et al., 2008). We show in this study that all of these enzymes were highly expressed in HCC and two of them (AKR1B10 and AKR1C3) were significantly upregulated in TCGA HCC specimens. The abundant expression of these enzymes and their deregulation in HCC may contribute to the development of cancer resistance to doxorubicin-containing HCC chemotherapeutic regimens (Le Grazie et al., 2017).

It has been proposed that the process of absorption, distribution, metabolism, and excretion of xenobiotics (e.g., drugs and dietary constituents) is coordinated in the liver mainly through the coregulation of ADME genes by transcription factors (Congiu et al., 2009) (Fig. 12). The direct evidence 


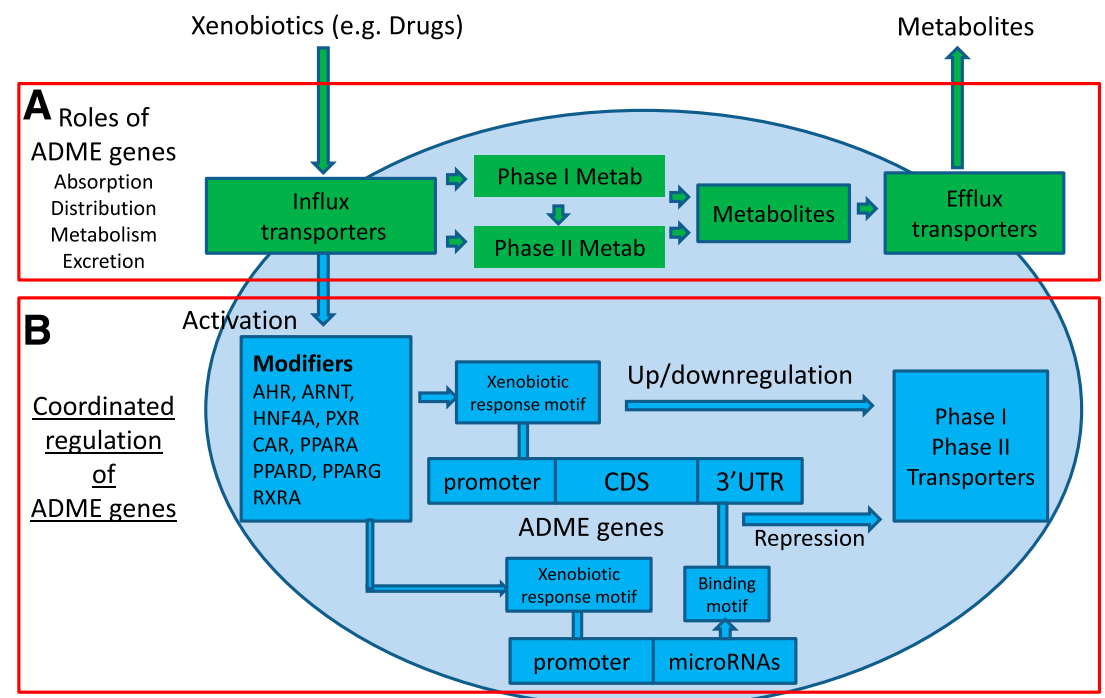

Fig. 12. Coordinated regulation of ADME genes in the liver (hepatocytes) and its deregulation in HCC. (A) It is believed that the process of absorption, distribution, metabolism, and excretion of xenobiotics (e.g., drugs and dietary constituents) is coordinated efficiently in the liver upon exposure to endogenous and exogenous compounds such as dietary constituents and therapeutic drugs. (B) This coordinated process is mainly mediated through the coregulation of ADME genes by ADME modifiers, including liver-enriched (e.g., HNF4A, PPARA, PPARD, and PPARG) and xenobiotic-activated (e.g., AHR, PXR, and CAR) transcription factors. The deregulation of these transcription factors, miRNAs, and epigenetics (e.g., hypermethylation) lead to wide spread deregulation of ADME genes in HCC.

Hepatocyte

to support this hypothesis came from the discoveries that a large number of ADME genes (e.g., CYP, $U G T, A B C$, and $S L C$ transporters) are induced (rarely repressed) by ligandactivated transcription factors (e.g., PXR, FXR, CAR, PPAR, AhR, and Nrf2) upon exposure to xenobiotics (e.g., drugs) (Scotto, 2003; Chen et al., 2013; Zanger and Schwab, 2013; Hu et al., 2014a). Further indirect evidence to support this hypothesis came from the studies that have shown positive correlation of the mRNA levels between ADME genes and transcription factors such as PXR, CAR, and HNF4A in normal (Slatter et al., 2006; Wortham et al., 2007) and nonHCC diseased (Congiu et al., 2002, 2009; Kurzawski et al., 2012) liver tissues. However, these correlation studies mainly relied on quantitative real-time polymerase chain reaction to measure gene expression levels and thus only analyzed a limited number of ADME genes. In this study, a comprehensive expression correlation analysis of the TCGA HCC RNAseq data showed for the first time that most core ADME genes were significantly positively correlated with each other. We further showed for the first time that the expression levels of about one-half of the core ADME genes were significantly positively (AHR, ARNT, HNF4A, PXR, CAR, PPARA, and $R X R A)$ or negatively ( $P P A R D$ and $P P A R G$ ) correlated with those of the nine transcription factors that are classified as ADME modifiers. Collectively, these observations provide compelling evidence supporting a coordinated hepatic regulation of the broader ADME transcriptome. Moreover, this study suggests that the coordinate regulation model of ADME genes may also extend to post-transcriptional regulation by miRNAs (Fig. 12); however, further work will be required to define the ADME gene/miRNA regulatory network.

In conclusion, this study reveals a widespread deregulation of ADME genes in HCC and widespread expression correlations of ADME genes to each other and with transcription factors. The deregulation of ADME genes represents part of the de-differentiation of liver function in HCC and is likely due to deregulation of epigenetics, transcription factors, and miRNAs. The coregulation of ADME genes mediated by liver-enriched (e.g., HNF4A, PPARA, PPARD, and PPARG) and xenobiotic-activated (e.g., AHR, PXR, CAR, and NRF2) transcription factors ensures that the process of absorption, distribution, metabolism, and excretion is coordinated efficiently upon exposure to endogenous and exogenous compounds such as dietary constituents and therapeutic drugs (Fig. 12).

\section{Authorship Contributions}

Participated in research design: Hu, Marri, McKinnon, Mackenzie, Meech.

Conducted experiments: $\mathrm{Hu}$, Marri.

Performed data analysis: Hu, Marri, Meech.

Wrote or contributed to the writing of the manuscript: $\mathrm{Hu}$, Meech, Marri, Mackenzie, McKinnon.

\section{References}

Andrisani OM, Studach L, and Merle P (2011) Gene signatures in hepatocellular carcinoma (HCC). Semin Cancer Biol 21:4-9.

Blanchard RL, Freimuth RR, Buck J, Weinshilboum RM, and Coughtrie MW (2004) A proposed nomenclature system for the cytosolic sulfotransferase (SULT) superfamily. Pharmacogenetics 14:199-211.

Borel F, Han R, Visser A, Petry H, van Deventer SJ, Jansen PL, and Konstantinova P; Réseau Centre de Ressources Biologiques Foie (French Liver Biobanks Network), France (2012) Adenosine triphosphate-binding cassette transporter genes up-regulation in untreated hepatocellular carcinoma is mediated by cellular microRNAs. Hepatology 55:821-832.

Bruix J, Qin S, Merle P, Granito A, Huang YH, Bodoky G, Pracht M, Yokosuka O, Rosmorduc O, Breder V, et al.; RESORCE Investigators (2017) Regorafenib for patients with hepatocellular carcinoma who progressed on sorafenib treatment (RESORCE): a randomised, double-blind, placebo-controlled, phase 3 trial. Lancet 389:56-66.

Chen H, Shen ZY, Xu W, Fan TY, Li J, Lu YF, Cheng ML, and Liu J (2014) Expression of P450 and nuclear receptors in normal and end-stage Chinese livers. World J Gastroenterol 20:8681-8690.

Chen QX, Hu HH, Zhou Q, Yu AM, and Zeng S (2013) An overview of ABC and SLC drug transporter gene regulation. Curr Drug Metab 14:253-264.

Chen X, Cheung ST, So S, Fan ST, Barry C, Higgins J, Lai KM, Ji J, Dudoit S, Ng IO, et al. (2002) Gene expression patterns in human liver cancers. Mol Biol Cell 13 1929-1939.

Chen Y, Zeng L, Wang Y, Tolleson WH, Knox B, Chen S, Ren Z, Guo L, Mei N, Qian F, et al. (2017) The expression, induction and pharmacological activity of CYP1A2 are post-transcriptionally regulated by microRNA hsa-miR-132-5p. Biochem Pharmacol 145:178-191.

Cho MY, Cheong JY, Lim W, Jo S, Lee Y, Wang HJ, Han KH, and Cho H (2014) Prognostic significance of catalase expression and its regulatory effects on hepatitis $\mathrm{B}$ virus $\mathrm{X}$ protein $(\mathrm{HBx})$ in $\mathrm{HBV}$-related advanced hepatocellular carcinomas. Oncotarget 5:12233-12246.

Chou YY, Cheng AL, and Hsu HC (1997) Expression of P-glycoprotein and p53 in advanced hepatocellular carcinoma treated by single agent chemotherapy: clinical correlation. J Gastroenterol Hepatol 12:569-575.

Chow RKK, Tsz-Kwan Sin S, Liu M, Li Y, Man Chan TH, Song Y, Chen L, Lai-Wan Kwong D, and Guan XY (2016) AKR7A3 suppresses tumorigenicity and chemoresistance in hepatocellular carcinoma through attenuation of ERK, c-Jun and NF- $\kappa$ B signaling pathways. Oncotarget 8:83469-83479. 
Congiu M, Mashford ML, Slavin JL, and Desmond PV (2002) UDP glucuronosyltransferase mRNA levels in human liver disease. Drug Metab Dispos 30:129-134. Congiu M, Mashford ML, Slavin JL, and Desmond PV (2009) Coordinate regulation of metabolic enzymes and transporters by nuclear transcription factors in human liver disease. J Gastroenterol Hepatol 24:1038-1044.

Dean M, Rzhetsky A, and Allikmets R (2001) The human ATP-binding cassette (ABC) transporter superfamily. Genome Res 11:1156-1166.

Edenberg HJ (2007) The genetics of alcohol metabolism: role of alcohol dehydrogenase and aldehyde dehydrogenase variants. Alcohol Res Health 30:5-13.

Farazi PA and DePinho RA (2006) Hepatocellular carcinoma pathogenesis: from genes to environment. Nat Rev Cancer 6:674-687.

Fisher CD, Lickteig AJ, Augustine LM, Ranger-Moore J, Jackson JP, Ferguson SS, and Cherrington NJ (2009) Hepatic cytochrome P450 enzyme alterations in humans with progressive stages of nonalcoholic fatty liver disease. Drug Metab Dis pos 37:2087-2094.

Fung KM, Samara EN, Wong C, Metwalli A, Krlin R, Bane B, Liu CZ, Yang JT, Pitha JV, Culkin DJ, et al. (2006) Increased expression of type $23 \alpha$-hydroxysteroid dehydrogenase/type $517 \beta$-hydroxysteroid dehydrogenase (AKR1C3) and its relationship with androgen receptor in prostate carcinoma. Endocr Relat Cancer 13:169-180.

Ghassabian S, Rawling T, Zhou F, Doddareddy MR, Tattam BN, Hibbs DE, Edwards RJ, Cui PH, and Murray M (2012) Role of human CYP3A4 in the biotransformation of sorafenib to its major oxidized metabolites. Biochem Pharmacol 84:215-223.

Guichard C, Amaddeo G, Imbeaud S, Ladeiro Y, Pelletier L, Maad IB, Calderaro J, Bioulac-Sage P, Letexier M, Degos F, et al. (2012) Integrated analysis of somatic mutations and focal copy-number changes identifies key genes and pathways in hepatocellular carcinoma. Nat Genet 44:694-698.

Hardwick RN, Ferreira DW, More VR, Lake AD, Lu Z, Manautou JE, Slitt AL, and Cherrington NJ (2013) Altered UDP-glucuronosyltransferase and sulfotransferase expression and function during progressive stages of human nonalcoholic fatty liver disease. Drug Metab Dispos 41:554-561.

Hardwick RN, Fisher CD, Canet MJ, Lake AD, and Cherrington NJ (2010) Diversity in antioxidant response enzymes in progressive stages of human nonalcoholic fatty liver disease. Drug Metab Dispos 38:2293-2301.

Hardwick RN, Fisher CD, Canet MJ, Scheffer GL, and Cherrington NJ (2011) Variations in ATP-binding cassette transporter regulation during the progression of human nonalcoholic fatty liver disease. Drug Metab Dispos 39:2395-2402.

Hayes JD and Dinkova-Kostova AT (2014) The Nrf2 regulatory network provides an interface between redox and intermediary metabolism. Trends Biochem Sci 39:199-218.

Hediger MA, Clémençon B, Burrier RE, and Bruford EA (2013) The ABCs of membrane transporters in health and disease (SLC series): introduction. Mol Aspects Med 34:95-107.

Heise M, Lautem A, Knapstein J, Schattenberg JM, Hoppe-Lotichius M, Foltys D, Weiler N, Zimmermann A, Schad A, Gründemann D, et al. (2012) Downregulation of organic cation transporters OCT1 (SLC22A1) and OCT3 (SLC22A3) in human hepatocellular carcinoma and their prognostic significance. BMC Cancer 12:109.

Higuchi T, Todaka H, Sugiyama Y, Ono M, Tamaki N, Hatano E, Takezaki Y, Hanazaki K, Miwa T, Lai S, et al. (2016) Suppression of microRNA-7 (miR-7) biogenesis by nuclear factor 90-nuclear factor 45 complex (NF90-NF45) controls cell proliferation in hepatocellular carcinoma. J Biol Chem 291:21074-21084.

Ho DW, Kai AK, and Ng IO (2015) TCGA whole-transcriptome sequencing data reveals significantly dysregulated genes and signaling pathways in hepatocellular carcinoma. Front Med 9:322-330.

Hu DG, Meech R, McKinnon RA, and Mackenzie PI (2014a) Transcriptional regulation of human UDP-glucuronosyltransferase genes. Drug Metab Rev 46:421-458.

Hu DG, Rogers A, and Mackenzie PI (2014b) Epirubicin upregulates UDP glucuronosyltransferase $2 \mathrm{~B} 7$ expression in liver cancer cells via the p53 pathway. $\mathrm{Mol}$ Pharmacol 85:887-897.

Innocenti F, Iyer L, Ramírez J, Green MD, and Ratain MJ (2001) Epirubicin glucuronidation is catalyzed by human UDP-glucuronosyltransferase 2B7. Drug Metab Dispos 29:686-692.

Jahn SC, Solayman MH, Lorenzo RJ, Langaee T, Stacpoole PW, and James MO (2016) GSTZ1 expression and chloride concentrations modulate sensitivity of cancer cells to dichloroacetate. Biochim Biophys Acta 1860:1202-1210.

Jin B, Wang W, Du G, Huang GZ, Han LT, Tang ZY, Fan DG, Li J, and Zhang SZ (2015a) Identifying hub genes and dysregulated pathways in hepatocellular carcinoma. Eur Rev Med Pharmacol Sci 19:592-601.

Jin S, Chen J, Chen L, Histen G, Lin Z, Gross S, Hixon J, Chen Y, Kung C, Chen Y, et al. (2015b) ALDH2(E487K) mutation increases protein turnover and promotes murine hepatocarcinogenesis. Proc Natl Acad Sci USA 112:9088-9093.

Jin Y, Yu D, Tolleson WH, Knox B, Wang Y, Chen S, Ren Z, Deng H, Guo Y, and Ning B (2016) MicroRNA hsa-miR-25-3p suppresses the expression and drug induction of CYP2B6 in human hepatocytes. Biochem Pharmacol 113:88-96.

Kamarajah SK, Frankel TL, Sonnenday C, Cho CS, and Nathan H (2018) Critical evaluation of the American Joint Commission on Cancer (AJCC) 8th edition staging system for patients with hepatocellular carcinoma (HCC): a surveillance, epidemiology, end results (SEER) analysis. J Surg Oncol 117:644-650.

Kassner N, Huse K, Martin HJ, Gödtel-Armbrust U, Metzger A, Meineke I, Brockmöller J, Klein K, Zanger UM, Maser E, et al. (2008) Carbonyl reductase 1 is a predominant doxorubicin reductase in the human liver. Drug Metab Dispos 36: $2113-2120$

Kurzawski M, Dziedziejko V, Post M, Wójcicki M, Urasińska E, Miętkiewski J, and Droździk M (2012) Expression of genes involved in xenobiotic metabolism and transport in end-stage liver disease: up-regulation of $A B C C 4$ and $C Y P 1 B 1$. Pharmacol Rep 64:927-939.

Lee JE, Bae SH, Choi JY, Yoon SK, You YK, and Lee MA (2014) Epirubicin, cisplatin, 5 -FU combination chemotherapy in sorafenib-refractory metastatic hepatocellular carcinoma. World J Gastroenterol 20:235-241.

Lee JS, Chu IS, Heo J, Calvisi DF, Sun Z, Roskams T, Durnez A, Demetris AJ, and Thorgeirsson SS (2004) Classification and prediction of survival in hepatocellular carcinoma by gene expression profiling. Hepatology 40:667-676.
Le Grazie M, Biagini MR, Tarocchi M, Polvani S, and Galli A (2017) Chemotherapy for hepatocellular carcinoma: the present and the future. World J Hepatol 9 907-920.

Li Y, Wan D, Wei W, Su J, Cao J, Qiu X, Ou C, Ban K, Yang C, and Yue H (2008) Candidate genes responsible for human hepatocellular carcinoma identified from differentially expressed genes in hepatocarcinogenesis of the tree shrew (Tupaia belangeri chinesis). Hepatol Res 38:85-95.

Lin HK, Steckelbroeck S, Fung KM, Jones AN, and Penning TM (2004) Characterization of a monoclonal antibody for human aldo-keto reductase AKR1C3 (type $23 \alpha$-hydroxysteroid dehydrogenase/type $517 \beta$-hydroxysteroid dehydrogenase); immunohistochemical detection in breast and prostate. Steroids 69:795-801.

Lin S, Hoffmann K, and Schemmer P (2012) Treatment of hepatocellular carcinoma a systematic review. Liver Cancer 1:144-158.

Liu CJ, Yang JH, Huang FZ, Nie WP, Liu CP, Mao XH, Yin XM, Shen XB, Peng C, Chen MF, et al. (2017) Glutathione-s-transferase A 4 (GSTA4) suppresses tumor growth and metastasis of human hepatocellular carcinoma by targeting AKT pathway. Am J Transl Res 9:301-315.

Llovet JM and Hernandez-Gea V (2014) Hepatocellular carcinoma: reasons for phase III failure and novel perspectives on trial design. Clin Cancer Res 20:2072-2079.

Llovet JM, Ricci S, Mazzaferro V, Hilgard P, Gane E, Blanc JF, de Oliveira AC, Santoro A, Raoul JL, Forner A, et al.; SHARP Investigators Study Group (2008) Sorafenib in advanced hepatocellular carcinoma. $N$ Engl J Med 359:378-390.

Love MI, Huber W, and Anders S (2014) Moderated estimation of fold change and dispersion for RNA-seq data with DESeq2. Genome Biol 15:550

Lu L, Zhou J, Shi J, Peng XJ, Qi XX, Wang Y, Li FY, Zhou FY, Liu L, and Liu ZQ (2015) Drug-metabolizing activity, protein and gene expression of UDPglucuronosyltransferases are significantly altered in hepatocellular carcinoma patients. PLoS One 10:e0127524.

Marchitti SA, Brocker C, Stagos D, and Vasiliou V (2008) Non-P450 aldehyde oxidizing enzymes: the aldehyde dehydrogenase superfamily. Expert Opin Drug Metab Toxicol 4:697-720.

Matkowskyj KA, Bai H, Liao J, Zhang W, Li H, Rao S, Omary R, and Yang GY (2014) Aldoketoreductase family 1B10 (AKR1B10) as a biomarker to distinguish hepatocellular carcinoma from benign liver lesions. Hum Pathol 45:834-843.

Matsunaga T, Wada Y, Endo S, Soda M, El-Kabbani O, and Hara A (2012) Aldo-keto reductase $1 \mathrm{~B} 10$ and its role in proliferation capacity of drug-resistant cancers. Front Pharmacol 3:5.

Mikkaichi T, Suzuki T, Onogawa T, Tanemoto M, Mizutamari H, Okada M, Chaki T, Masuda S, Tokui T, Eto N, et al. (2004) Isolation and characterization of a digoxin transporter and its rat homologue expressed in the kidney. Proc Natl Acad Sci USA 101:3569-3574.

Miners JO, Chau N, Rowland A, Burns K, McKinnon RA, Mackenzie PI, Tucker GT, Knights KM, and Kichenadasse G (2017) Inhibition of human UDPglucuronosyltransferase enzymes by lapatinib, pazopanib, regorafenib and sorafenib: implications for hyperbilirubinemia. Biochem Pharmacol 129:85-95.

Mohri T, Nakajima M, Fukami T, Takamiya M, Aoki Y, and Yokoi T (2010) Human CYP2E1 is regulated by miR-378. Biochem Pharmacol 79:1045-1052.

Moustafa MA, Ogino D, Nishimura M, Ueda N, Naito S, Furukawa M, Uchida T, Ikai I, Sawada H, and Fukumoto M (2004) Comparative analysis of ATP-binding cassette $(\mathrm{ABC})$ transporter gene expression levels in peripheral blood leukocytes and in liver with hepatocellular carcinoma Cancer Sci 95:530-536.

Nakano M, Fukushima Y, Yokota S, Fukami T, Takamiya M, Aoki Y, Yokoi T, and Nakajima M (2015) CYP2A7 pseudogene transcript affects CYP2A6 expression in human liver by acting as a decoy for miR-126. Drug Metab Dispos 43:703-712

Nelson DR, Zeldin DC, Hoffman SM, Maltais LJ, Wain HM, and Nebert DW (2004) Comparison of cytochrome P450 (CYP) genes from the mouse and human genomes, including nomenclature recommendations for genes, pseudogenes and alternativesplice variants. Pharmacogenetics 14:1-18.

Novotna R, Wsol V, Xiong G, and Maser E (2008) Inactivation of the anticancer drugs doxorubicin and oracin by aldo-keto reductase (AKR) 1C3. Toxicol Lett 181:1-6.

Nwosu ZC, Megger DA, Hammad S, Sitek B, Roessler S, Ebert MP, Meyer C, and Dooley S (2017) Identification of the consistently altered metabolic targets in human hepatocellular carcinoma. Cell Mol Gastroenterol Hepatol 4:303-323.e1.

Obaidat A, Roth M, and Hagenbuch B (2012) The expression and function of organic anion transporting polypeptides in normal tissues and in cancer. Annu Rev Pharmacol Toxicol 52:135-151.

Ohya H, Shibayama Y, Ogura J, Narumi K, Kobayashi M, and Iseki K (2015) Regorafenib is transported by the organic anion transporter 1B1 and the multidrug resistance protein 2. Biol Pharm Bull 38:582-586.

Okabe H, Satoh S, Kato T, Kitahara O, Yanagawa R, Yamaoka Y, Tsunoda T, Furukawa Y, and Nakamura Y (2001) Genome-wide analysis of gene expression in human hepatocellular carcinomas using cDNA microarray: identification of genes involved in vira carcinogenesis and tumor progression. Cancer Res 61:2129-2137.

Papageorgiou I and Court MH (2017) Identification and validation of the microRNA response elements in the $3^{\prime}$-untranslated region of the UDP glucuronosyltransferase (UGT) $2 B 7$ and $2 B 15$ genes by a functional genomics approach. Biochem Pharmacol 146:199-213.

Park T, Yi SG, Shin YK, and Lee S (2006) Combining multiple microarrays in the presence of controlling variables. Bioinformatics 22:1682-1689.

Peer CJ, Sissung TM, Kim A, Jain L, Woo S, Gardner ER, Kirkland CT, Troutman SM, English BC, Richardson ED, et al. (2012) Sorafenib is an inhibitor of UGT1A1 but is metabolized by UGT1A9: implications of genetic variants on pharmacokinetics and hyperbilirubinemia. Clin Cancer Res 18:2099-2107.

Penning TM (2015) The aldo-keto reductases (AKRs): overview. Chem Biol Interact 234:236-246.

Rhodes DR, Kalyana-Sundaram S, Mahavisno V, Varambally R, Yu J, Briggs BB, Barrette TR, Anstet MJ, Kincead-Beal C, Kulkarni P, et al (2007) Oncomine 3.0 genes, pathways, and networks in a collection of 18,000 cancer gene expression profiles. Neoplasia 9:166-180. 
Richart J, Brunt EM, and Di Bisceglie AM (2002) Expression of P-glycoprotein and C-MOAT in human hepatocellular carcinoma: detection by immunostaining. Dig Dis Sci 47:2454-2458.

Rieger JK, Reutter S, Hofmann U, Schwab M, and Zanger UM (2015) Inflammationassociated microRNA-130b down-regulates cytochrome $\mathrm{P} 450$ activities and directly targets CYP2C9. Drug Metab Dispos 43:884-888.

Roessler S, Jia HL, Budhu A, Forgues M, Ye QH, Lee JS, Thorgeirsson SS, Sun Z Tang ZY, Qin LX, et al. (2010) A unique metastasis gene signature enables prediction of tumor relapse in early-stage hepatocellular carcinoma patients. Cancer Res 70:10202-10212.

Schaeffeler E, Hellerbrand C, Nies AT, Winter S, Kruck S, Hofmann U, van der Kuip $\mathrm{H}$, Zanger UM, Koepsell H, and Schwab M (2011) DNA methylation is associated with downregulation of the organic cation transporter OCT1 (SLC22A1) in human hepatocellular carcinoma. Genome Med 3:82.

Scotto KW (2003) Transcriptional regulation of ABC drug transporters. Oncogene 22: $7496-7511$

Shang L, Zhu G, Su H, Chen B, Ye X, Chen X, Xiao K, Li L, Peng M, and Peng T (2017) Identification of alcohol dehydrogenase as a potential prognostic marker in HBV-related hepatocellular carcinoma. Int J Clin Exp Med 10:4457-4472.

Shen J, Wang S, Zhang YJ, Kappil M, Wu HC, Kibriya MG, Wang Q, Jasmine F, Ahsan H, Lee PH, et al. (2012) Genome-wide DNA methylation profiles in hepatocellular carcinoma. Hepatology 55:1799-1808.

Shi Y, Liu Y, Wei Z, Zhang Y, Zhang L, Jiang S, Xiong Y, Shen L, He L, Xing Q, et al. (2015) Hsa-miR-27a is involved in the regulation of CYP3A4 expression in human livers from Chinese Han population. Pharmacogenomics 16:1379-1386.

Skarka A, Skarydová L, Stambergová H, and Wsól V (2011) Anthracyclines and their metabolism in human liver microsomes and the participation of the new microsomal carbonyl reductase. Chem Biol Interact 191:66-74.

Slatter JG, Templeton IE, Castle JC, Kulkarni A, Rushmore TH, Richards K, He Y, Dai X, Cheng OJ, Caguyong M, et al. (2006) Compendium of gene expression profiles comprising a baseline model of the human liver drug metabolism transcriptome. Xenobiotica 36:938-962.

Swift B, Nebot N, Lee JK, Han T, Proctor WR, Thakker DR, Lang D, Radtke M, Gnoth MJ, and Brouwer KL (2013) Sorafenib hepatobiliary disposition: mechanisms of hepatic uptake and disposition of generated metabolites. Drug Metab Dispos 41:1179-1186.

Thurnherr T, Mah WC, Lei Z, Jin Y, Rozen SG, and Lee CG (2016) Differentially expressed miRNAs in hepatocellular carcinoma target genes in the genetic information processing and metabolism pathways. Sci Rep 6:20065.

Tsuboyama T, Onishi H, Kim T, Akita H, Hori M, Tatsumi M, Nakamoto A, Nagano H, Matsuura N, Wakasa K, et al. (2010) Hepatocellular carcinoma: hepatocyteselective enhancement at gadoxetic acid-enhanced MR imaging-correlation with expression of sinusoidal and canalicular transporters and bile accumulation. $R a$ diology 255:824-833.

Vander Borght S, Libbrecht L, Blokzijl H, Faber KN, Moshage H, Aerts R, Van Steenbergen W, Jansen PL, Desmet VJ, and Roskams TA (2005) Diagnostic and pathogenetic implications of the expression of hepatic transporters in focal lesions occurring in normal liver. J Pathol 207:471-482.

van Erp NP, Gelderblom H, and Guchelaar HJ (2009) Clinical pharmacokinetics of tyrosine kinase inhibitors. Cancer Treat Rev 35:692-706.

Vavricka SR, Jung D, Fried M, Grützner U, Meier PJ, and Kullak-Ublick GA (2004) The human organic anion transporting polypeptide 8 (SLCO1B3) gene is transcriptionally repressed by hepatocyte nuclear factor 3beta in hepatocellular carcinoma. J Hepatol 40:212-218.

Wei RR, Zhang MY, Rao HL, Pu HY, Zhang HZ, and Wang HY (2012) Identification of $\mathrm{ADH} 4$ as a novel and potential prognostic marker in hepatocellular carcinoma. Med Oncol 29:2737-2743.

Wlcek K and Stieger B (2014) ATP-binding cassette transporters in liver. Biofactors 40:188-198.

Woo HG, Choi JH, Yoon S, Jee BA, Cho EJ, Lee JH, Yu SJ, Yoon JH, Yi NJ, Lee KW, et al. (2017) Integrative analysis of genomic and epigenomic regulation of the transcriptome in liver cancer. Nat Commun 8:839.

Wortham M, Czerwinski M, He L, Parkinson A, and Wan YJ (2007) Expression of constitutive androstane receptor, hepatic nuclear factor $4 \alpha$, and P450 oxidoreductase genes determines interindividual variability in basal expression and activity of a broad scope of xenobiotic metabolism genes in the human liver. Drug Metab Dispos 35:1700-1710.

Wurmbach E, Chen YB, Khitrov G, Zhang W, Roayaie S, Schwartz M, Fiel I, Thung S, Mazzaferro V, Bruix J, et al. (2007) Genome-wide molecular profiles of HCVinduced dysplasia and hepatocellular carcinoma. Hepatology 45:938-947.

Xie C, Yan TM, Chen JM, Li XY, Zou J, Zhu LJ, Lu LL, Wang Y, Zhou FY, Liu ZQ, et al. (2017) LC-MS/MS quantification of sulfotransferases is better than conventional immunogenic methods in determining human liver SULT activities: implication in precision medicine. Sci Rep 7:3858.

$\mathrm{Xu} \mathrm{C}$, Li CY, and Kong AN (2005) Induction of phase I, II and III drug metabolism/transport by xenobiotics. Arch Pharm Res 28:249-268.

Yan T, Gao S, Peng X, Shi J, Xie C, Li Q, Lu L, Wang Y, Zhou F, Liu Z, et al. (2015a) Significantly decreased and more variable expression of major CYPs and UGTs in liver microsomes prepared from HBV-positive human hepatocellular carcinoma and matched pericarcinomatous tissues determined using an isotope label-free UPLC-MS/MS method. Pharm Res 32:1141-1157.

Yan T, Lu L, Xie C, Chen J, Peng X, Zhu L, Wang Y, Li Q, Shi J, Zhou F, et al. (2015b) Severely impaired and dysregulated cytochrome P450 expression and activities in hepatocellular carcinoma: implications for personalized treatment in patients. Mol Cancer Ther 14:2874-2886.

Yang CK, Wang XK, Liao XW, Han CY, Yu TD, Qin W, Zhu GZ, Su H, Yu L, Liu XG, et al. (2017) Aldehyde dehydrogenase 1 (ALDH1) isoform expression and potential clinical implications in hepatocellular carcinoma. PLoS One 12:e0182208.

Ye L, Yang X, Guo E, Chen W, Lu L, Wang Y, Peng X, Yan T, Zhou F, and Liu Z (2014) Sorafenib metabolism is significantly altered in the liver tumor tissue of hepatocellular carcinoma patient. PLoS One 9:e96664.

Yu D, Green B, Marrone A, Guo Y, Kadlubar S, Lin D, Fuscoe J, Pogribny I, and Ning B (2015a) Suppression of CYP2C9 by microRNA hsa-miR-128-3p in human liver cells and association with hepatocellular carcinoma. Sci Rep 5:8534.

Yu D, Green B, Tolleson WH, Jin Y, Mei N, Guo Y, Deng H, Pogribny I, and Ning B (2015b) MicroRNA hsa-miR-29a-3p modulates CYP2C19 in human liver cells. Biochem Pharmacol 98:215-223.

Zanger UM and Schwab M (2013) Cytochrome P450 enzymes in drug metabolism: regulation of gene expression, enzyme activities, and impact of genetic variation. Pharmacol Ther 138:103-141.

Zhang K, Mu L, Ding MC, Xu R, Ding ZJ, and Liang J (2018) NF $\kappa$ B mediated elevation of KCNJ11 promotes tumor progression of hepatocellular carcinoma through interaction of lactate dehydrogenase A. Biochem Biophys Res Commun 495:246-253.

Zhang SY, Surapureddi S, Coulter S, Ferguson SS, and Goldstein JA (2012) Human CYP2C8 is post-transcriptionally regulated by microRNAs 103 and 107 in human liver. Mol Pharmacol 82:529-540.

Zhang YJ, Chen Y, Ahsan H, Lunn RM, Chen SY, Lee PH, Chen CJ, and Santella RM (2005) Silencing of glutathione $S$-transferase $P 1$ by promoter hypermethylation and its relationship to environmental chemical carcinogens in hepatocellular carcinoma. Cancer Lett 221:135-143.

Zhong S, Han W, Hou C, Liu J, Wu L, Liu M, Liang Z, Lin H, Zhou L, Liu S, et al. (2017) Relation of transcriptional factors to the expression and activity of cytochrome P450 and UDP-glucuronosyltransferases 1A in human liver: co-expression network analysis. AAPS J 19:203-214.

Zhong S, Tang MW, Yeo W, Liu C, Lo YM, and Johnson PJ (2002) Silencing of GSTP1 gene by $\mathrm{CpG}$ island DNA hypermethylation in HBV-associated hepatocellular carcinomas. Clin Cancer Res 8:1087-1092.

Zimmerman EI, Hu S, Roberts JL, Gibson AA, Orwick SJ, Li L, Sparreboom A, and Baker SD (2013) Contribution of OATP1B1 and OATP1B3 to the disposition of sorafenib and sorafenib-glucuronide. Clin Cancer Res 19:1458-1466.

Zollner G, Wagner M, Fickert P, Silbert D, Fuchsbichler A, Zatloukal K, Denk H, and Trauner M (2005) Hepatobiliary transporter expression in human hepatocellular carcinoma. Liver Int 25:367-379.

Address correspondence to: Dr. Dong Gui Hu, Department of Clinical Pharmacology and Flinders Centre for Innovation in Cancer, Flinders University College of Medicine and Public Health, Flinders Medical Centre, Bedford Park, South Australia, Australia. E-mail: donggui.hu@flinders.edu.au 